九州大学学術情報リポジトリ

Kyushu University Institutional Repository

\title{
Pelecypod Fauna of the Shimajiri Group in Miyako-jima, Okinawa Prefecture, Japan
}

Sato, Yoshio

Faculty of Sciences, Kyushu University

Masuda, Koichiro

Department of Geology, Miyagi University of Education

Shuto, Tsugio

Kyushu University : Emeritus Professor

https://doi.org/10.5109/1526112

出版情報：九州大學理學部紀要：Series D, Geology. 26 (1)，pp.1-49，1986-01-20. Faculty of Science, Kyushu University バージョン：

権利関係 : 
Mem. Fac. Sci., Kyushu Univ., Ser. D, Geol., Vol. XXVI, No. 1, pp. 1-49, text-figs. $1-2$, table 1 , plates 1-5, January 20, 1986

\title{
Pelecypod Fauna of the Shimajiri Group in Miyako-jima, Okinawa Prefecture, Japan
}

\author{
Yoshio SATO, Kôichirô MASUdA* and Tsugio SHUTO**
}

\begin{abstract}
The present paper is the first report on the paleontological study of the molluscan fauna (Pelecypoda) from the Shimajiri Group in Miyako-jima and is divided into two parts. Outline of the geology of Miyako-jima, stratigraphical position of fossil localities, occurrence of fossils and correlation of the molluscan fauna are included in the first part. Systematic description of the fauna is to be given in the second part. The molluscan fauna from the Shimajiri Group in Miyako-jima is correlated with those of the Shimajiri Group in Okinawa-jima, the Miyazaki Group and the Kakegawa Group in Southwest Japan. Fifty-three species are described and 3 species and 1 subspecies are new to science.
\end{abstract}

\section{Introduction}

The Shimajiri Group developed in Miyako-jima of the Ryukyu Islands, Southwest Japan, has been stratigraphically studied by DOAN, PASEUR and Fosberg (1960), UJiIE and OKi (1974), Nakagawa, Nirtsuma, Murakami and WATANABE (1976), YAZAKI (1978), YAZAKI and OYAMA (1979, 1980), and NAKAMORI (1982). While the geological age of that group has hitherto been assigned to the Late Miocene to Pleistocene based upon planktonic foraminifers by HUANG (1968) and UJIIE and OKI (1974), and based upon calcareous nannoplanktons by NISHIDA (1973), it has been considered to be Pliocene in age based upon planktonic foraminifers and paleomagnetism together by KAMEyama (1975) and Nakagawa, Nirtsuma, Murakami and Watanabe (1976). As pointed in OGASAWARA and MASUDA (1983), nothing has been done on molluscs.

The present writers collected numerous fossil molluscs from the Shimajiri Group at several localities in Miyako-jima. Examination of those molluscs gives interesting knowledge on the faunal relation with the Kakegawa fauna of Southwest Japan. The present paper deals with the pelecypods and the result of gastropods and a few scaphopods will be published at later date.

* Department of Geology, Miyagi University of Education.

** Emeritus Professor of Kyushu University.

Manuscript received August 10, 1985. 


\section{Part 1 Stratigraphy and Pelecypod Fauna}

\section{Outline of the Stratigraphy}

The Shimajiri Group mainly cropping out along the northeastern coast of Miyako-jima is classified into the following formations in upward sequence (NAKAMORI, 1982).

Oura Formation:-This formation corresponds with the Ikema Sandstone and the Ogami Sandstone of DoAN, Paseur and FosBerg (1960), the Nanseien Formation of UJIIE and OKI (1974) and the Ogami-jima Formation of YAZAKI (1978) and YAZAKI and OYAMA (1979).

The lower part of this formation consisting of conglomerate and crossbedded sandstone crops out in the Ogami-jima. The upper part consisting of sandstone and alternation of sandstone and siltsone is distributed at north of Shimajiri and its environs in Miyako-jima. Molluscan shells are found from Ogami-jima (Locality 1) and north of Shimajiri (Locality 2).

Oonogoshi Formation:-This formation consists of massive siltstone and corresponds with a part of the Gusukube Shale of DOAN, PASEUR and FosBerg (1960), the lower part of Yonahama Mudstone of UJIIE and OKI (1974) and the lower part of the Gusukube Formation of YAZAKI (1978) and YAZAKI and OYAMA (1980). Many molluscan shells are collected from northwest of Piseogazaki (Locality 3) and Piseogazaki (Locality 4).

Yonahama Formation:-The Nobaru Formation of DoAN, PASEUR and FOSBERG (1960), the upper part of the Yonahama Mudstone of UJIIE and OKI (1974), and the upper part of the Gusukube Formation, Higa conglomerate of YAZAKI (1978) and YAZAKI and OYAMA (1980) correspond with this formation consisting of tuffaceous siltstone, tuff and conglomerate. Numerous fossil molluscs are collected from the conglomerate developed at northeast of Higa, Gusukube-cho (Locality 5).

Minebari Formation:-This formation corresponds with the upper part of the Gusukube Formation of DoAN, PASEUR and FosBerg (1960), the Minebari alternation of UJIIE and OKI (1974) and the Henna Formation of YAZAKI (1978) and YAZAKI and OYAMA (1980), and consists of alternation of sandstone and siltstone. Some fossil molluscs have been recorded (NAKAMORI, 1982), but the writers did not collect any molluscs.

\section{Occurrence of fossils and remarks on Fossil Fauna}

The results of field observation are briefly described in the following lines. Fossil localities are five and their locations are shown in Fig. 1. Precise descriptions of fossil localities are listed in the separate chapter.

1) Locality 1:-Oogami-jima (ôura Formation).

Some molluscan shells occur in conglomeratic cross-bedded sandstone and siltstone which are irregularly in association with thin lignite seams. The re- 

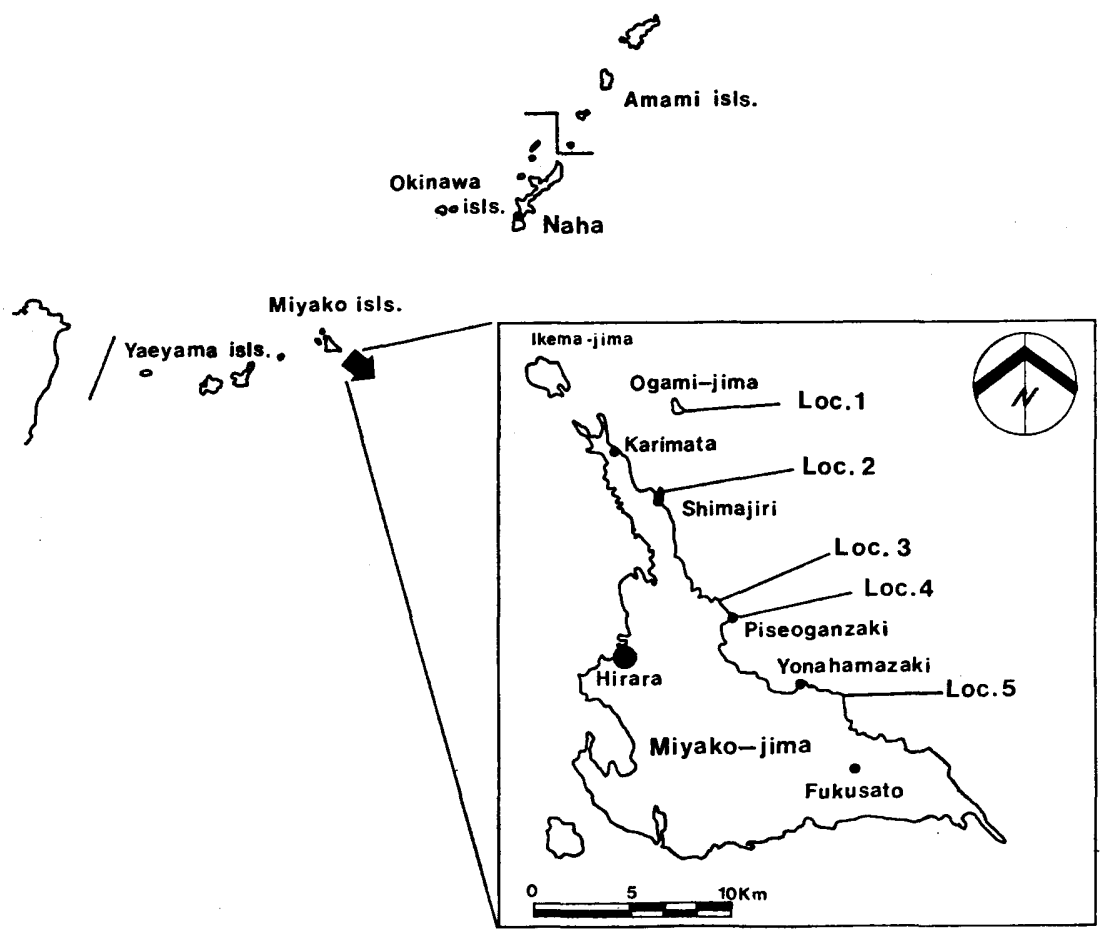

Fig. 1. Map showing the fossil localities of the Shimajiri Group in Miyako-jima.

presentative species is Crassostrea gigas (THUNBERG). The association of Crassostrea gigas, Cyclina sp. cf. (C. sinensis (GMELIN)) and Telescopium sp. suggests that a brackish environment existed during deposition in the Ogamijima area.

2) Locality 2:-Shimajiri (ôura Formation).

Molluscan shells occur sporadically and are orientated nearly parallel with bedding planes in the well sorted very fine sandstone in association with simple corals and rarely with echinoids. The representative species are Amusium pleuronectes okinawaensis $\mathrm{n}$. subsp. which occurs as conjointed valves and is accompanied by small isolated shells of Chlamys (Mimachlamys) satoi (YokoYAMA).

3) Locality 3:--Northwest of Piseoganzaki (Oonogoshi Formation).

Molluscan shells consisting of isolated and somewhat fragmental pelecypods are densely crowded in lenticular medium-grained sandstone intercalated in siltstone. Among the molluscs Chlamys (Mimachlamys) satoi and Anomia chinensis PHILIPPI are the representative species.

4) Locality 4:-Piseoganzaki (Oonogoshi Formation).

Molluscs occur sporadically and are orientated nearly parallel with bedding plane in the siltstone. Small shells of Amusium pleuronectes okinawaensis n. subsp. and Glycymeris rotunda (DUNKER) are the representative species from this locality. They usually occur as conjoined valves. 
Loc. 2

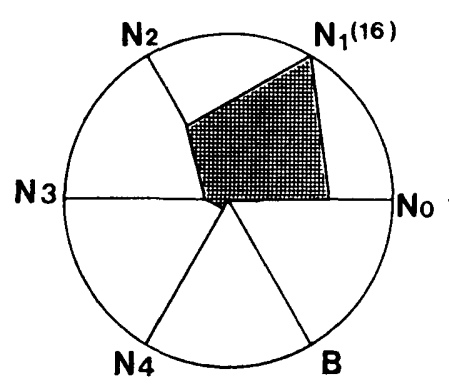

Loc. 3

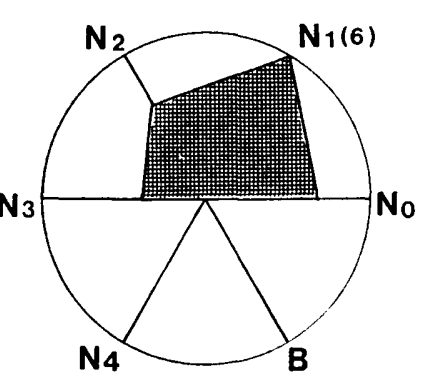

Loc. 4

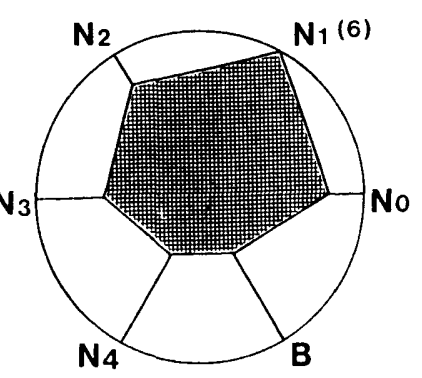

Loc. 5

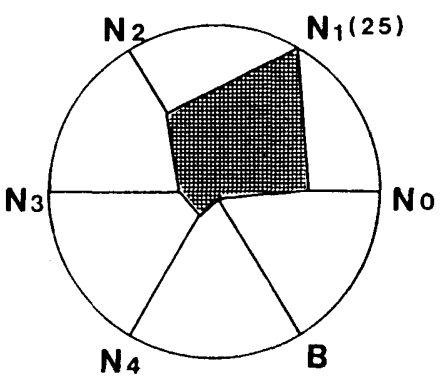

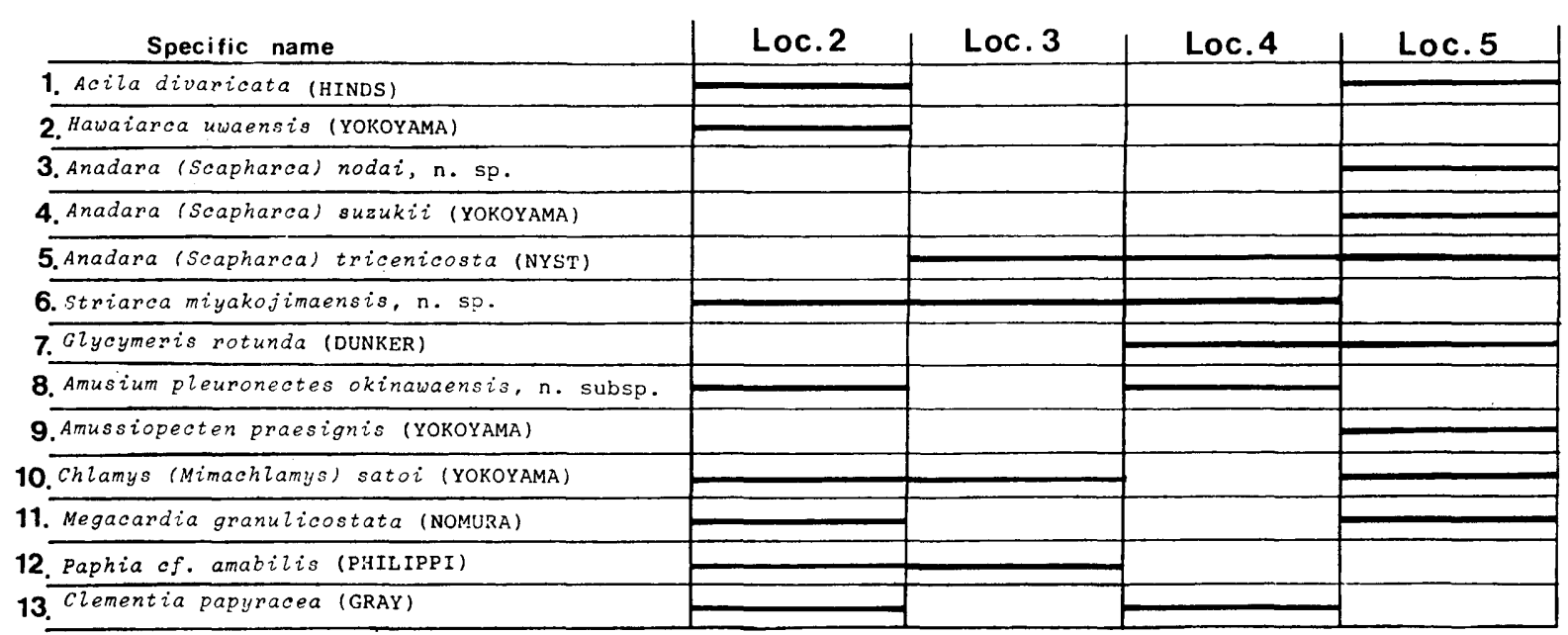

Fig. 2. Vertical distribution of the living representatives of the pelecypod species and stratigraphical ranges of important index fossils. Each fossil locality is arranged in ascending order. Parenthesized figures are the total number of species. 


\section{5) Locality 5:-Higa (Yonahama Formation).}

Molluscs comprising many water worn shells are densely crowded in the andesitic pebbly conglomerate. Among them Glycymeris rotunda is the most representative species.

Recent species from the Shimajiri Group on Miyako-jima is 26 and 65 percent of the total number of species. As shown in Fig. 2, living representatives of the pelecypod species from the Shimajiri Group in Miyako-jima are generally living in a depth zone from tidal $\mathrm{N}_{0}$ to mesoneritic $\mathrm{N}_{2}$ (between 20-30 and 50-60 m) in their vertical distribution. Chlamys (Mimachlamys) satoi (YokoYAMA) and Amussiopecten praesignis (YoKoYAMA) found at Loc. 2 to Loc. 5 are the important indices for the Pliocene in South Japan.

\section{Correlation and Geological Age}

The complete faunal list is presented in Table 1. Fifty-three species were distinguished among the molluses obtained from the Oura, Oonogoshi and Yonahama Formations and among them 3 species and 1 subspecies are found to be new to science. Chlamys (Mimachlamys) satoi (YokoYAMA) ranging from the ôra Formation to Yonahama Formation in the Miyako-jima is stratigraphically very important species and also Anadara sedanensis (MARTIN), Anadara suzukii (YoKочAмA), Amussiopecten praesignis (YoKоYAMA), etc. are also regarded as stratigraphical indices. As they have been known only from the Pliocene formations in the southern Japanese Islands and Taiwan, it is evident that the geological age of the Shimajiri Group is Early Pliocene in a twofold classification. On the basis of faunal assemblages the Shimajiri Group in Miyako-jima is reasonably correlated with the upper part of the Shimajiri Group in Okinawajima, Tajima Formation in Tanega-shima, Kagoshima Prefecture, the Miyazaki Group in Miyazaki Prefecture, the Tonohama Group in Kochi Prefecture, the Kakegawa Group in Shizuoka Prefecture and the Byoritsu Formation in Taiwan.

\section{Depository of specimens}

All the specimens described in the present paper are preserved and registered in the Department of Geology, Faculty of Science, Kyushu University.

\section{List of fossil localities}

Loc. 1 (Ôgami-jima):-Sea cliff, about $100 \mathrm{~m}$ east of ôgami, Hirara City, ôgami-jima, Okinawa Prefecture. oura Formation (Long. $125^{\circ} 22^{\prime} 12^{\prime \prime} \mathrm{E}$, Lat. $\left.24^{\circ} 59^{\prime} 11^{\prime \prime} \mathrm{N}\right)$.

Loc. 2 (Shimajiri) :-Sea cliff, about $200 \mathrm{~m}$ north of Shimajiri, Hirara City, Miyako-jima, Okinawa Prefecture. Ôura Formation (Long. $125^{\circ} 23^{\prime} 12^{\prime \prime} \mathrm{E}$, Lat. $24^{\circ} 52^{\prime} 23^{\prime \prime} \mathrm{N}$ ).

Loc. 3 (Northwest of Piseoganzaki):-Sea cliff, northwest of Piseoganzaki, about $1 \mathrm{~km}$ northeast of Fukuyama, Hirara City, Miyako-jima, Okinawa 
Table 1. List of fossil pelecypods from the Shimajiri Group in Miyako-jima.

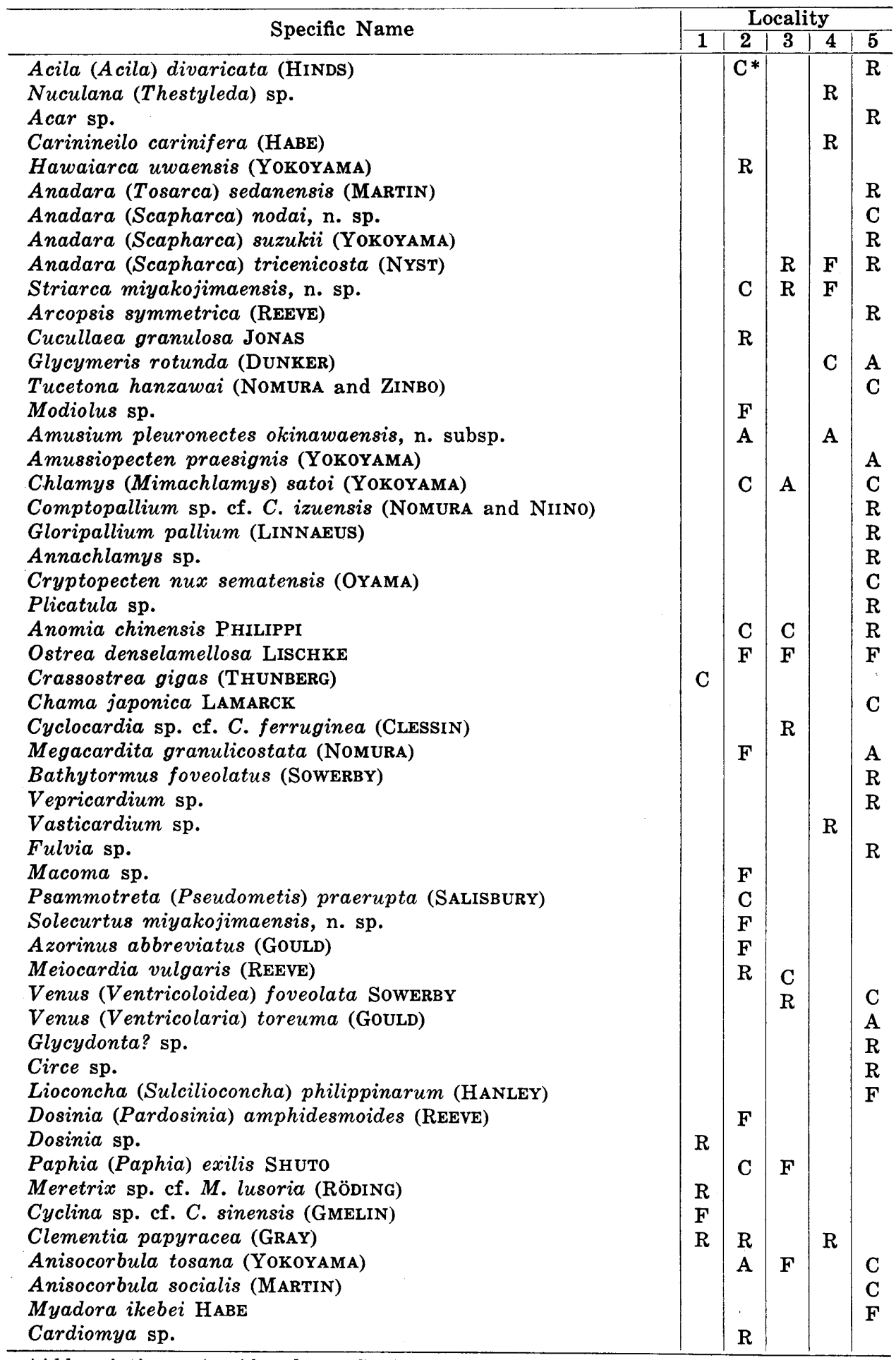

*Abbreviation: A-Abundant; C-Common; F-Few; R-Rare. 
Prefecture. Oonogoshi Formation (Long. $125^{\circ} 24^{\prime} 7^{\prime \prime} \mathrm{E}$, Lat. $24^{\circ} 48^{\prime} \mathrm{N}$ ).

Loc. 4 (Piseoganzaki) :-Sea cliff at Piseoganzaki, about $1.5 \mathrm{~km}$ east of Fukuyama, Hirara City, Miyako-jima Okinawa Prefecture. Oonogoshi Formation (Long. $125^{\circ} 21^{\prime} 11^{\prime \prime} \mathrm{E}$, Lat. $24^{\circ} 49^{\prime} 4^{\prime \prime} \mathrm{N}$ ).

Loc. 5 (Higa) :-Sea cliff, about $2 \mathrm{~km}$ northeast of Higa, Gusukube-cho, Miyako-jima, Okinawa Prefecture. Yonahama Formation (Long. $125^{\circ} 23^{\prime 21}$ "E, Lat. $\left.24^{\circ} 46^{\prime} 52^{\prime \prime} \mathrm{N}\right)$.

\section{References cited (geology and biostratigraphy)}

Doan, D. B., PAseur, J. E. and Fosberg, F. R. (1960) : Military Geology of the Miyako archipelago, Ryukyu Retto (translated into Japanese by SUzUKI, K., 1973, Geological Data of Okinawa Prefecture), 1, 35-83.

Hanzawa, S. (1935): Topography and Geology of the Riukiu Islands. Sci. Rep., Tohoku Imp. Univ., [2], 17, 1-61.

HuANG, T. (1968) : Smaller Foraminifera from Miyako-jima, Ryukyu. Sci. Rep., Tohoku Univ., [2], 40, 47-63.

KameYama, T. (1975): Smaller Foraminiferas from Ogami-jima Ryukyu Islands. Earth Science (Jour. of the Assoc. for the Geol. Coll. in Japan, Chikyukagaku), 29, (6), 275-297.

Nakagawa, H., Nirtsuma, N., Murakami, M. and Watanabe, T. (1976) : Preliminary report of Magnetostratigraphy of the Shimajiri Group in Miyako-jima and Kumejima (in Japanese). Geological studies of the Ryukyu Islands, 1, 55-64.

NAKAMORI, T. (1982) : Geology of Miyako Gunto, Ryukyu Islands, Japan (in Japanese with English abstract). Contr. Inst. Geol. Paleont., Tohoku Univ., (84), 23-39.

NishidA, S. (1973) : Preliminary study of the Upper Cenozoic calcareous nannoplankton assemblages from the Nansei Islands (in Japanese with English abstract). Mem. Geol. Soc. Japan, (8), 65-75, pls. 1-2.

Ogasawara, K. and Masuda, K. (1983): Notes on the Paleoenvironments based upon the Cenozoic molluses in the Ryukyu Islands (in Japanese with English abstract). Mem. Geol. Soc. Japan, (22), 95-105.

UJIIE, H. and OKI, K. (1974): Uppermost Miocene-Lower Pleistocene, Planktonic Foraminifera from Shimajiri Group of Miyakojima. Mem. Natn. Sci. Mus., (7), 31-52.

YAZAKI, K. (1978): About the Shimajiri Group of Miyako-jima, Ryukyu Islands (in Japanese with English abstract). Geological studies of Ryukyu Islands, (3), 81-88.

and OYama, K. (1979): Geology of the Miyakojima-Hokubu District. Qardrangle Series Scale 1:50000, Miyakojima (in Japanese with English abstract), 19, (3), 1-43.

- $-(1980):$ Geology of Miyakojima District. Ibid., 19, (4), 41-83.

\section{Appendix}

Alphabetical index of place names with Japanese writing.
1. Fukuyama (福山)
2. Gusukube（城辺）
3. Higa (比嘉)
4. Minebari (嶺原)
5. Hirara (平良)
6. Ikema-jima (池間島)
7. Nanseien (南静園)
8. Ôgami-jima (大神島)
9. Ôonogoshi (大野越)
10. Oura（大浦）
11. Piseoganzaki (平瀬尾願崎)
12. Shimajiri (島尻)
13. Yonahama (与那浜) 


\title{
Part 2 Systematic description
}

\author{
Kôichirô MASUDA, Yoshio SATO and Tsugio SHUTO \\ Family Nuculidae \\ Genus Acila H. and A. AdAms, 1858 \\ Acila (Acila) divaricata (HINDS, 1843) \\ Pl. 1, Figs. 1, 2
}

1860. Nucula divaricata Hinds. HANLEY in Sowerby, Thes. Conch., vol. 3, p. 155, pl. 230, fig. 151.

1920. Nucula mirabilis AdAMS and Reeve. Yokohama, Jour. Coll. Sci., Imp. Univ. Tokyo, vol. 39 , art. 6 , p. 180 , pl. 19, figs. 9 a-c.

1936. Acila (Acila) divaricata. Schenck, Geol. Soc. Amer., Spec. Paper, no. 4, p. 90 , pl. 15, figs. 1-10, text-figs. $8(1,2)$.

1954. Acila divaricata. Taki and Oyama, Palaeont. Soc. Japan, Spec. Paper, no. 2, pl. 20, figs. 9a-c.

1958. Acila divaricata. HABE, Publ. Seto Mar. Biol. Lab., vol. 6, no. 3, p. 243, pl. 12, fig. 8.

1959. Acila (Acila) divaricata. IWAI, Bull. Fac. Educ., Hirosaki Univ., no. 5, p. 53, pl. 2, fig. 13.

1965. Acila divaricata. Kaseno and MatsuUra, Sci. Rep., Kanazawa Univ., vol. 10 , no. 1 , pl. 7 , fig. 8 .

1966. Acila divaricata. Sakagami et al., Jour. Hokkaido Univ. Educ., Ser. B, vol. 17 , no. 1 , pl. 2 , fig. 14.

1973. Acila (Acila) divaricata. Oyama, Palaeont. Soc. Japan, Spec. Paper, no. 17, p. 75, pl. 20 , fig. 25.

1977. Acila (Acila) divaricata. HABE, Syst. Moll. Japan, p. 15, pl. 2, fig. 5.

1980. Acila (Acila) divaricata. NodA, Sci. Rep. Inst. Geosci., Univ. Tsukuba, vol. 1 , p. 65 , pl. 1 , figs. $5,10 \mathrm{a}-\mathrm{b}$.

Remarks:-The present specimens are characterized by their mediumsized, moderately inflated shell with rostral sinus, distinct divaricate sculpture on surface, prominent teeth, anteriorly protruded deep resilifer and fine marginal serration and identical to Acila (Acila) divaricata (HINDS).

Acila submirabilis MAKIYAMA (1926) described from the Miocene Heirokudo Formation in North Korea resembles the present species, but submirabilis can be distinguished from the present one by its very small escutcheon, rounded anterior margin and smaller number of anterior teeth.

The present species has been illustrated from the Miocene formations in Central to Northern Japan (AoKI, 1954; ARAKI, 1960; TANAKA, 1960; MASUdA and TAKEGAWA, 1965; IWAI, 1965), but as the Miocene specimens are usually unfavorably preserved, further detailed study of them is necessary to settle this problem.

$\begin{array}{lcccrrr}\text { Dimensions } & \text { (in mm) :- } & & & & \\ \text { Valve } & \text { Intact } & \text { Intact } & \text { Intact } & \text { Right } & \text { Left } & \text { Left } \\ \text { Height } & 18.4 & 18.0 & 15.0 & 15.5 & 18.0 & 15.2 \\ \text { Length } & 24.5 & 23.3 & 19.6 & 21.0 & 23.2 & 20.0 \\ \text { Depth } & 13.7 & 11.8 & 10.7 & 6.0 & 6.9 & 5.8\end{array}$


Occurrence:-Loc. 2 (Common*); Loc. 5 (Rare*).

Geologic distribution:-Nojima Formation, Kanagawa Prefecture; Higashimeya Formation, Aomori Prefecture; Omma Formation, Ishikawa Prefecture; Tomikawa Formation, Hokkaido; Shinzato Formation, Okinawa Prefecture.

Recent distribution:-Honshu, Shikoku, Kyushu, Japan Sea and East China sea.

Geologic range:-Pliocene to Recent.

Family Malletiidae

Genus Carinineilo KURODA and HABE, 1971

Carinineilo carinifera (HABE, 1951)

Pl. 1, Fig. 3

1951. Malletia (Neilo) carinifera HABE. Gen. Japan. Shells, no. 1, p. 22, figs. $16,17$.

1971. Carinineilo carinifera. Kuroda, Habe and Oyama, Sea Shells Sagami Bay, p. 505 , pl. 66 , fig. 2 .

Remarks:-One rather well preserved specimen was examined. Shell small in size, thin, elliptical, slightly inflated; surface smooth but with rather distinct concentric growth lines near ventral margin and with two rather distinct carinae running from beak to posterior and anterior corners.

Dimensions (in $\mathrm{mm}$ ):-Height 7.5, length 10.0, depth ca. 3.0.

Occurrence:-Loc. 4 (Rare).

Recent distribution:-Honshu (Boso Peninsula as north limit) and Shikoku. Geologic range:-Pliocene to Recent.

\author{
Family Nuculanidae \\ Genus Nuculana LiNK, 1807 \\ Subgenus Thestyleda IREDALE, 1929 \\ Nuculana (Thestyleda) sp. \\ Pl. 1, Fig. 4
}

Remarks:-One imperfect specimen was examined. The present taxodont specimen is characterized by its two ridges running from beak to posterior corner and somewhat lamellated coarse concentric cords on surface and is reasonably included in Thestyleda. Further comparison can not be done because of imperfect preservation.

Dimensions (in $\mathrm{mm}$ ):-Height 6.5, length 12.0, depth ca. 3.5.

Occurrence:-Loc. 4 (Rare). * Abundant: more than ten specimens, Common: more than five specimens, Few: more
than two specimens, Rare: only one specimen. 


\author{
Family Arcidae \\ Genus Acar GRAY, 1857 \\ Acar sp.
}

Remarks:-One fragmental specimen was examined. Shell small in size and with distinct network by distinct radial threads and concentric growth lines.

Occurrence:-Loc. 5 (Rare).

Genus Hawaiarca DALL, BARTSCH and REHDER, 1938

Hawaiarca uwaensis (YoKoYAMA, 1928)

Pl. 1, Fig. 5

1928. Arca uwaensis Yokoyama. Jour. Fac. Sci., Imp. Univ. Tokyo, Ser. 2, vol. 2 , pt. 7 , p. 349 , pl. 67 , figs. $13,14$.

1935. "Bathyarca" uwaensis. OTUKA, Bull. Earthq. Res. Inst., Tokyo Imp. Univ., vol. 13 , pt. 4 , p. 884, pl. 55, figs. $114,115$.

1958. Hawaiarca uwaensis. HABE, Publ. Seto Mar. Biol. Lab., vol. 6, no. 3, p. 254, pl. 11, fig. 10.

1959. Hawaiarca uwaensis. MakiYama, Palaeont. Soc. Japan, Spec. Paper, no. 5 , pl. 65 , figs. 13,14 .

1966. Hawaiarca uwaensis. NodA, Sci. Rep., Tohoku Univ., 2nd Ser., vol. 38, no. 1 , p. 75 , pl. 10 , fig. 8 .

1971. Hawaiarca uwaensis. Kuroda, Habe and Oyama, Sea Shells Sagami Bay, p. 519 , pl. 67 , figs. 3,4 .

1980. Hawaiarca uwaensis. NodA, Sci. Rep. Inst. Geosci., Univ. Tsukuba, vol. 1, p. 72 , pl. 1, figs. $19 \mathrm{a}-\mathrm{b}, 20$.

Remarks:-One well preserved specimen was examined. The present specimen is characterized by its small, elongated quadrate shell with median depression and about 27 radial ribs which are somewhat granulate, broader than their interspaces and sometimes divide into two parts by a shallow longitudinal furrow near ventral margin. It quite conforms with Hawaiarca uwaensis (YokoYAMA).

In general features the present species resembles Hawaiarca nannodes (MARTIN, 1885) from Upper Miocene of Djakarta in Java, Indonesia, but $H$. nannodes can be distinguished from the present one by having a little larger and higher shell and less oblique posterior margin.

Dimensions (in $\mathrm{mm}$ ):-Height 5.4, length 10.0, depth ca. 3.5.

Occurrence:-Loc. 2 (Rare).

Geologic distribution:-Tokazan Formation, Taiwan; Nakoshi and Shinzato Formations, Okinawa Prefecture; Takanabe Member of Koyu Formation, Miyazaki Prefecture; Ninomiya and Naganuma Formations, Kanagawa Prefecture; Kiwada and Narita Formations, Chiba Prefecture; Hatsuzaki Formation, Ibaraki Prefecture.

Recent distribution:-Honshu (Boso Peninsula and Noto Peninsula as north limit), Shikoku and Kyushu.

Geologic range:-Pliocene to Recent. 


\author{
Genus Anadara GraY, 1847 \\ Subgenus Tosarca NoDA, 1965 \\ Anadara (Tosarca) sedanensis (MARTIN, 1910)
}

1910. Arca (Scapharca) sedanensis Martin. Samml. Geol. R. Mus., Leiden, $N$. F., Bd. 1, Heft 1, p. 381, pl. 54, fig. 125-127.

1942. Anadara (Scapharca) sedanensis. YABE and HATAI, Japan. Jour. Geol. Geogr., vol. 18, nos. 1-2, p. 73, pl. 7, fig. 9.

1966. Anadara (Tosarca) sedanensis. NobA, Sci. Rep., Tohoku Univ., 2nd Ser., vol. 38 , no. 1 , p. 123 .

Remarks:-One imperfect but well preserved specimen was examined. The present species is characterized by its large, subtrapezoidal, inflated shell, numerous, squarish, dichotomous, beaded radial ribs and depressed area along the posterior side.

Dimension (in $\mathrm{mm}$ ) :-Height 48.0 .

Occurrence:-Loc. 5 (Rare).

Distribution:-Middle Miocene Formations, Java; Pliocene Nakoshi Formation, Okinawa Prefecture; Byoritsu Formation, Taiwan.

Geologic range:-Pliocene.

Subgenus Scapharca GraY, 1847

Anadara (Scapharca) nodai MASUdA, SAto and ShUto, n. sp.

Pl. 1, Figs. 7a-b, 8, 9a-b, 10, 11a-b, 12

1965. Anadara (Scapharca?) sp. NodA, Trans. Proc. Palaeont. Soc. Japan, N. S., no. 59, p. 101, pl. 10, figs. $8,9$.

Holotype:-GK-L 11825.*

Type locality:-Loc. 5. Yonahama Formation.

Diagnosis:-Shell generally medium in size and oblong. Radial ribs stout, broader than their interspaces and its total number 20 to 22 . Ligamental area rather narrow. Ventral margin broadly rounded.

Description:-Shell medium in size, ovately oblong, rather thick, moderately inflated, longer than high, inequilateral; anterior side rounded, posterior side elongated, dorsal margin straight, depressed area along posterior side of shell, ventral margin broadly rounded. Surface with 20 to 22, elevated, smooth radial ribs which are more or less squarish in profile and broader than their interspaces having lamellated concentric incremental lines. Beak small, pointed and strongly incurved anteriorly; ligamental area rather narrow; teeth small, perpendicular to straight hinge line and slightly converge ventrally. Inner surface smooth except for fine longitudinal striations within pallial line and with distinct crenulations along ventral margin.

Comparison:-The present new species somewhat resembles Anadara ando $i$ (Nomura, 1933) described from the Byoritsu Formation, Taiwan, but it can be distinguished from andoi by its rather large oblong shell and radial ribs

* Abbreviation for Department of Geology, Kyushu University. 
broader than their interspaces. Also, Anadara (Scapharca) takaoensis (NoMURA, 1933) from the Kaizan Formation, Taiwan, can be distinguished from the present one by having smaller shell with about 25 radial ribs which are much broader than their interspaces and finely granulated on anterior part.

The present species also resembles $A$. hulshofi (MARTIN) from the lower Middle Miocene Rewbeng Bed of Java, but the former is distinguished from the latter in having somewhat stouter ribs of less number and more oblique posterior margin than the latter.

Dimensions (in $\mathrm{mm}$ ) :-

$\begin{array}{llllccc}\text { Valve } & \text { Left* } & \text { Left } & \text { Left } & \text { Right } & \text { Rignt } & \text { Right } \\ \text { Height } & 20.8 & 30.0 & 26.5 & 27.5 & 26.0 & 22.0 \\ \text { Length } & 41.0 & 40.0 & 38.0 & 42.5 & 37.0 & 33.0 \\ \text { Depth } & 14.5 & 14.5 & 13.5 & 14.5 & 11.0 & 10.0 \\ \text { * Holotype } & & & & & & \end{array}$

Occurrence:-Common.

Geologic distribution:-Ananai Formation, Kochi Prefecture.

Geologic range:-Pliocene.

Anadara (Scapharca) suzukii (YoкоYамA, 1926)

Pl. 1, Fig. 13

1926. Arca suzukii Yoкоуама. Jour. Fac. Sci., Imp. Univ. Tokyo, Ser. 2, vol. 1, pt. 9 , p. 368 , pl. 42 , figs. 6 , 7 .

1958. Anadara tricenicosta (NYST). MakiYama, Palaeont. Soc. Japan, Spec. Paper, no. 4, pl. 54, figs. 6, 7.

1965. Anadara (Scapharca) suzukii. NodA, Trans. Proc. Palaeont. Soc. Japan, N. S., no. 59 , p. 100 , pl. 10 , figs. $3-7,10-13$, pl. 11 , figs. $9,10$.

1966. Anadara (Scapharca) suzukii. NodA, Sci. Rep., Tohoku Univ., 2nd Ser., vol. 38 , no. 1 , p. 111 , pl. 8 , fig. 2.

1981. Anadara (Scapharca) suzukii. MASUda and Ogasawara, Mem. Vol. Prof. M. OMORI, pl. 2, fig. 1 .

Remarks:-The present species is characterized by its rather small shell having about 25, granulated, squarish radial ribs which are narrower than their interspaces with distinct lamellated concentric lines.

The present species was first described on the basis of the type specimen from the Tonohama Formation, Kochi Prefecture by YokoYama (1926) and then it was regarded as a synonym of Anadara (Scapharca) tricenicosta (NYST) by MAKryama (1958). However, it is evident that the present one can be distinguished from tricenicosta by its rather small shell and smaller number of granulated radial ribs.

Dimensions (in $\mathrm{mm}$ ):-Height 22.5, length 33.0, depth 10.5 .

Occurrence:-Loc. 5 (Few).

Geologic distribution:-Tonohama Formation, Kochi Prefecture; Dainichi Formation, Shizuoka Prefecture.

Geologic range:-Pliocene. 
Anadara (Scapharca) tricenicosta (NYST, 1848)

Pl. 1, Fig. 14

1844. Arca radiata ReEve. Conch. Icon., vol. 2, Arca, sp. 40, pl. 6, fig. 40.

1848. Arca tricenicosta Nyst. Mem. Acad. Roy. Belg., vol. 22, p. 74.

1882. Scapharca philippiana DUNKer. Index Moll. Mar. Japon, p. 236.

1891. Arca (Scapharca) philippiana. Kobelt in MARTini und Chemnitz, Syst. Conch. Cab., vol. 8, p. 90, pl. 25, figs. 1, 2.

1928b. Arca (Scapharca) philippiana. Yokоyama, Imp. Geol. Surv. Japan, Rep., no. 101 , p. 103 , pl. 17 , figs. 4,5 .

1960. Anadara (Scapharca) tricenicosta. Makiyama, Palaeont. Soc. Japan, Spec. Paper, no. 6, pl. 103, figs. 4, 5.

1965. Anadara (Scapharca) tricenicosta. NodA, Trans. Proc. Palaeont. Soc. Japan, N. S., no. 59, p. 102, pl. 10, figs. 1, 2, 16, 17.

1966. Anadara (Scapharca) tricenicosta. NodA, Sci. Rep., Tohoku Univ., 2nd Ser., vol. 38 , no. 1 , p. 113 , pl. 10, fig. 7 .

Remarks:-Several rather unfavorably preserved specimens were examined. The present species is characterized by its elongated shell and about 28, distinct, squarish, granulated, non-dichotomous radial ribs.

Occurrence:-Loc. 3. (Rare); Loc. 4 (Few); Loc 5 (Rare).

Geologic distribution:-Byoritsu Formation, Taiwan; Nakoshi Formation, Okinawa Prefecture; Naganuma Formation, Kanagawa Prefecture.

Recent distribution:-Honshu (Boso Peninsula as north limit), Shikoku, Kyushu, Korea, China, Philippines and Indonesia.

Geologic range:-Pliocene to Recent.

\section{Genus Striarca CoNRAD, 1862}

Striarca miyakojimaensis MASUDA, SATo and Shuto, n. sp.

Pl. 1, Figs. 15, 16a-b

Holotype:-GK-L 11826.

Type locality:-Loc. 2. Ôura Formation.

Diagnosis:-Shell generally small in size, ovate and moderately inflated. Ligamental area narrow and oblonged triangular. Internal surface striated but no marginal serration.

Description:-Shell small, ovate, medium in thickness, moderately inflated, subequilateral; ventral margin nearly parallel with hinge line. Beak small, pointed and strongly incurved. Surface sculptured with numerous, somewhat granulated, low radial ribs, intercalary threads and concentric incremental lines; intercalary threads usually appear near beak and tend to become nearly equal with radial ribs in strength near ventral margin. Ligamental area narrow, oblonged triangular. Hinge slightly curved, with small, numerous teeth. Interior surface radially striated but no marginal serration; adductor scars bordered with raised myophoric flange.

Comparison:-The present new species is closely related with Striarca sinensis (THIELE, 1931), a Recent species of the South China Sea, but the latter can be distinguished from the present one by its strongly inflated shell, prominent beak, wide ligamental area and internal marginal serration. 
The present species is also closely related with Striarca compressa (MARTIN, 1885) from Sonde Bed, East Java, but it differs therefrom in having larger number of radial ribs, intercalary threads and no internal marginal serration.

Dimensions (in $\mathrm{mm}$ ) :-

$\begin{array}{lcccccc}\text { Valve } & \text { Right* } & \text { Right } & \text { Right } & \text { Left } & \text { Left } & \text { Intact } \\ \text { Height } & 10.4 & 14.5 & 10.0 & 15.0 & 12.0 & 18.0 \\ \text { Length } & 15.4 & 19.0 & 14.2 & 21.0 & 17.0 & 23.0 \\ \text { Depth } & 3.8 & 5.0 & - & 5.0 & 4.0 & 10.0 \\ \text { * Holotype } & & & & & & \\ \\ \text { Occurrence:-Loc. } 2 \text { (Common) ; Loc. } 3 \text { (Rare); Loc. } 4 \text { (Few). }\end{array}$

Genus Arcopsis KoEneN, 1885

Arcopsis symmetrica (REEVE, 1844)

1844. Arca symmetrica ReEve. Conch. Icon., vol. 2, Arca, sp. 117, pl. 17, fig. 117.

1891. Arca (Arca) symmetrica. Kobelt in MARTini und Chemnitz, Syst. Conch. Cab., vol. 8, p. 94, pl. 25, figs. 7, 8 .

1853. Striarca (Galactella) symmetrica. HABE in KURODA, Illust. Cat. Japan. Shells, no. 25, p. 209, pl. 30, figs. 12, 13.

1858. Striarca (Galactella) symmetrica. Yамамото and HABE, Bull. Mar. Biol. Sta. Asamushi, Tohoku Univ., vol. 9, no. 1, p. 6, pl. 2, figs. 19, 20.

1961. Striarca (Galactella) symmetrica. HAYASAKA, Sci. Rep., Tohoku Univ., 2nd Ser., vol. 33, no. 1, p. 27, pl. 2, figs. 9a-b.

1966. Striarca oyamai. NoDA, Ibid., vol. 38 , no. 1 , p. 73 , pl. 4 , figs. $8,9$.

1966. Striarca symmetrica. NoDA, Ibid., vol. 38 , no. 1 , p. 73.

1969. Striarca symmetrica. IWAI and SIOBARA, Bull. Educ. Fac., Hirosaki Univ., no. 15 , pl. 1 , figs. $3 a-c$.

1973. Striarca symmetrica. ItolgaWA and OGAWA, Sci. Rep., Tohoku Univ., 2nd Ser., Spec. Vol., no. 6, p. 71, pl. 5, fig. 3.

1977. Arcopsis symmetrica. HABE, Syst. Moll. Japan, p. 41, pl. 7, figs. 11-14.

Remarks:-One fragmental specimen was examined. HABE (1953) described Striarca (Galactella) oyamai, n. sp. from Wakayama Prefecture and discriminated from Striarca symmetrica. But subsequently he (HABE, 1977) regarded oyamai as a synonym of symmetrica to represent a morphological type of inland seas of the latter.

Occurrence:-Loc. 5 (Rare).

Geologic distribution:-Sawane and Funabashi Formations, Niigata Prefecture; Dainichi Formation, Shizuoka Prefecture; Takamatsu and Sakishima Formations, Aichi Prefecture; Naganuma and Shimosueyoshi Formations, Kanagawa Prefecture; Umegase, Narita Mandano and Numa Formations, Chiba Prefecture; Yoshida Formation, Kagoshima Prefecture; Hiratoko Formation, Ishikawa Prefecture; Nakoshi Formation, Okinawa Prefecture.

Recent distribution:-Honshu (Boso Peninsula as north limit), Shikoku Kyushu, Korea, China, Taiwan, Philippines and East Indies.

Geologic range:-Pliocene to Recent. 


\author{
Family Cucullaeidae \\ Genus Cucullaea LAMARCK, 1801 \\ Cucullaea granulosa JoNAS, 1846
}

Pl. 1, Fig. 17

1869. Cucullaea granulosa JoNAS. REEve, Conch. Icon., vol. 17, Cucullaea, sp. 2, pl. 1, figs. 2a-b.

1891. Cucullaea concamerata Martini. Kobelt in Martini und Chemnitz, Syst. Conch. Cab., vol. 8, p. 5, pl. 1, figs. 3, 4, pl. 5, figs. $1,2$.

1891. Cucullaea granulosa. Kobelt in MarTini und Chemnitz, Ibid., p. 6, pl. 1, fig. 5.

1926. Cucullaea concamerata. Yoкоуама, Jour. Fac. Sci., Imp. Univ. Tokyo, Ser. 2 , vol. 1 , pt. 9 , p. 360 , pl. 41 , fig. 2.

1928. Cucullaea pamotanensis MarTiN. YokoYama, Imp. Geol. Surv. Japan, Rep., no. 101, p. 107, pl. 18, figs. 4, 5.

1929. Cucullaea concamerata. YokоYAмA, Ibid., no. 104, p. 17, pl. 8, fig. 2.

1930. Cucullaea (labiata SolANDER, var. ?) granulosa. KUROdA, Venus, vol. 2, no. 1 , append., p. 34 , fig. 40.

1933. Cucullaea granulosa. Nomura, Sci. Rep., Tohoku Imp. Univ., 2nd Ser., vol. 16 , no. 1 , p. 44 , pl. 4 , fig. 2 .

1960. Cucullea granulosa. MakiYama, Palaeont. Soc., Japan, Spec. Paper, no. 6, pl. 104, figs. 4, 4a, 5, 5a.

1960. Cucullaea granulosa. MakiYama, Ibid., no. 6, pl. 115, fig. 2.

1964. Cucullaea labiata granulosa. HABE, Bull. Nat. Sci. Mus., vol. 7, no. 3, p. 261, figs. $1,2$.

1971. Cucullaea labiosa granulosa. KURODA, HABE and OYAMA, Sea Shells Sagami Bay, p. 516, pl. 70, figs. 1, 2.

1977. Cucullaea labiosa granulosa. HABE, Syst. Moll. Japan, p. 43, pl. 7, figs. $1,2$.

Remarks:-One imperfect specimen was examined. This species is characterized by its inflated shell with obtuse ridge running from beak to postero ventral corner, numerous, flat, smooth radial threads which tend to be divided into two parts by a shallow longitudinal furrow towards ventral margin and fine concentric lines forming fine reticulation.

Occurrence:-Loc. 2 (Rare).

Geologic distribution:-Dainichi Formation, Shizuoka Prefecture; Tonohama Formation, Kochi Prefecture; Byoritsu Formation, Taiwan.

Recent distribution:-Honshu (Boso Peninsula as north limit), Shikoku, Kyushu China and Malaysia.

Geologic range:-Pliocene to Recent.

Family Glycymerididae

Genus Glycymeris da Costa, 1778

Glycymeris rotunda (DUNKER, 1882)

Pl. 1, Figs. 18, 19, 20, 21

1882. Pectunculus rotundus Dunker. Index Moll. Mar. Japon., p. 236, pl. 16, figs. 9, 10.

1889. Pectunculus rotundus. PILSBRY, Cat. Japan. Mar. Moll. p. 150. 
1920. Pectunculus nipponicus Yokoуама. Jour. Coll. Sci., Imp. Univ. Tokyo, vol. 39 , art. 6 , p. 168 , pl. 18, figs. 3-7.

1922. Pectunculus yamakawai YokоYAмA. Ibid., vol. 44, art. 1, p. 190, pl. 16, figs. 4,5 .

1927. Glycymeris rotunda. MakiYama, Mem. Coll. Sci., Kyoto Imp. Univ., Ser. $B$, vol. 1 , no. 1 , p. 31 , pl. 1 , fig. 7 .

1954. Glycymeris rotunda. TAKI and OYAMA, Palaeont. Soc. Japan, Spec. Paper, no. 2 , pl. 36 , figs. 4,5 .

1962. Glycymeris rotunda. Hayasaka, Trans. Proc. Palaeont. Soc. Japan, N. S., no. 47 , pl. 45 , figs. 8,9 .

1971. Glycymeris rotunda. Kuroda, HABE and Oyama, Sea Shells Sagami Bay, p. 532, pl. 71 , fig. 6 .

1973. Glycymeris rotunda. OYAMA, Palaeont. Soc. Japan, Spec. Paper, no. 17, p. 77 , pl. 22 , figs. 5,6 .

Remarks:-The present specimen is characterized by the following features:-Shell medium to small in size, thick, inequilateral, moderately inflated. Beak small, pointed. Anterior dorsal margin rounded, shorter than broadly rounded posterior dorsal margin. Surface with rather coarse concentric growth lines and numerous, faint, fine radial threads. Interior surface smooth, but pallial line and adductor scars distinct; distinct serration at ventral margin; ligamental area triangular in shape, with distinct divaricate sculpture.

The present species shows a great variation in shell form. Usually shells are suborbicular in younger stage, but shells tend to become longer than high with growth.

Comparison:-This species can be distinguished from Glycymeris vestita (DUNKER), a Recent species of Japan, by its much smaller, posteriorly protruded shell and faint, fine radial furrows. Also, Glycymeris yessoensis (SowERBY), Recent species of Northern Japan, can be distinguished from the present one by its large, less inflated shell, not prominent beak and rather distinct radial furrows.

\section{Explanation of Plate 1}

(All figures in natural size unless otherwise stated)

Page

Figs. 1, 2. Acila (Acila) divaricata (HINDs). Loc. 2. ............ 8

Fig. 3. Carinineilo carinifera ( $\mathrm{HABE}) . \times 3.5$. Loc. $4 . \ldots \ldots \ldots \ldots \ldots \ldots$

Fig. 4. Nuculana (Thestyleda) sp. Loc. 4. ................... 9

Fig. 5. Hawaiarca uwaensis (YokoYAMA) $\times 2$. Loc. $2 . \ldots \ldots \ldots \ldots \ldots$

Fig. 6. Anadara (Tosarca) sedanensis (MARTIN). Loc. 5. ..............11

Figs. 7a-b, 8, 9a-b, 10, 11a-b, 12. Anadara (Scapharca) nodai MASUdA,

SAto and Shuto, n. sp., 9a-b, Holotype, Reg. No. GK-L 11825. Loc. 5. . . . 11

Fig. 13. Anadara (Scapharca) suzukii (YokоYAмA). Loc. 5. ...........12

Fig. 14. Anadara (Scapharca) tricenicosta (NYST). Loc. 5. ...........13

Fig. 15, 16a-b. Striarca miyakojimaensis MASUda, SATo and ShUto, n.

sp., 15, ×2., 16a-b, Holotype, Reg. No. GK-L 11826. $\times 1.5$. Loc. 2. . . . . . 13

Fig. 17. Cucullaea granulosa JoNAS. Loc. 2. ......................15

Figs. 18, 19, 20, 21. Glycymeris rotunda (DUNkeR). Loc. 5. .........15

Figs. 22a-b. Tucetona hanzawai (NomURA and $\mathrm{ZINBO}_{\mathrm{N}}$ ). $\times 2$. Loc. 5. ......17

Fig. 23. Modiolus sp. Loc. 2. .......................... 


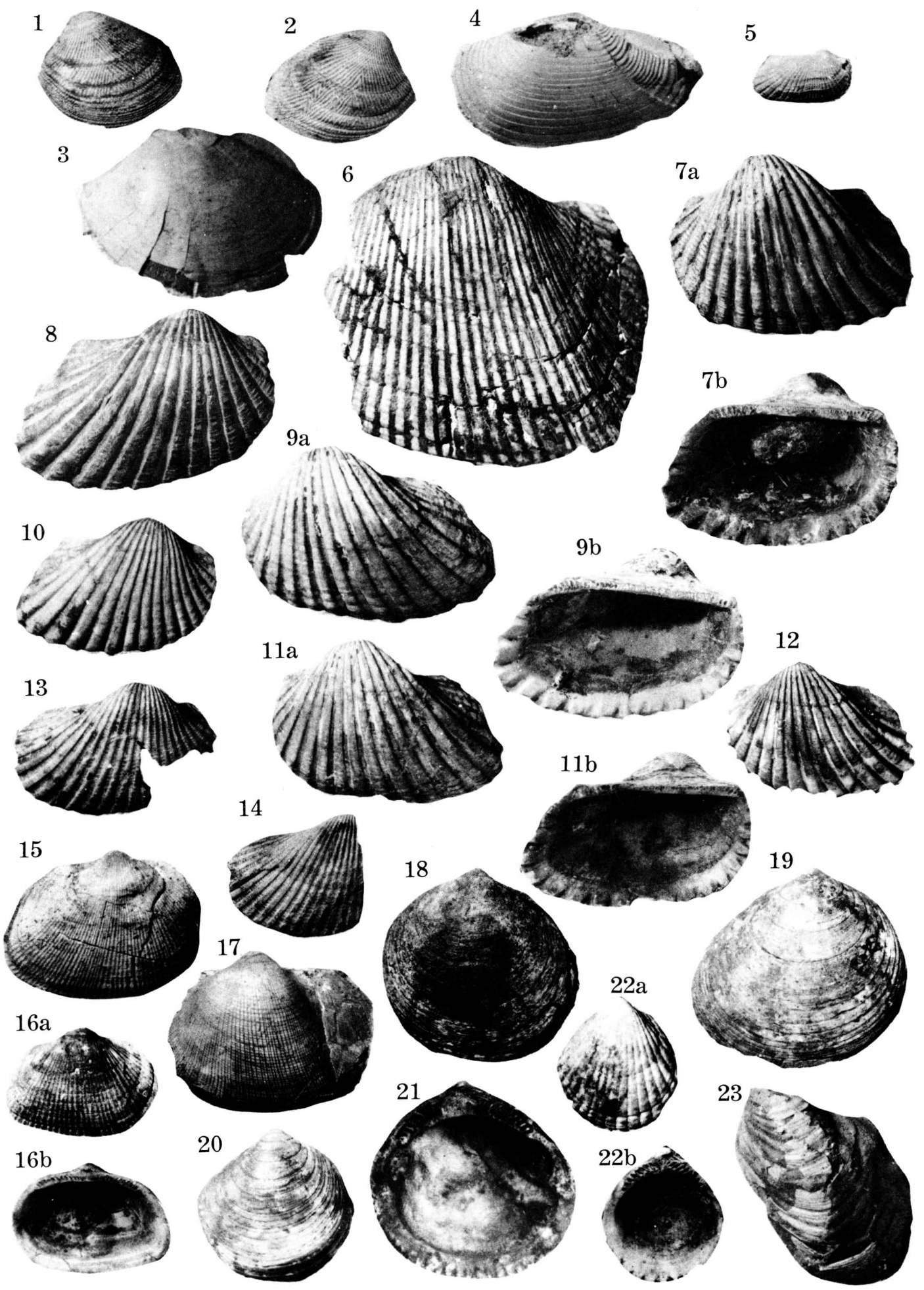

Y. SAto et al.: Pelecypod Fauna of the Shimajiri Group in Miyako-jima 


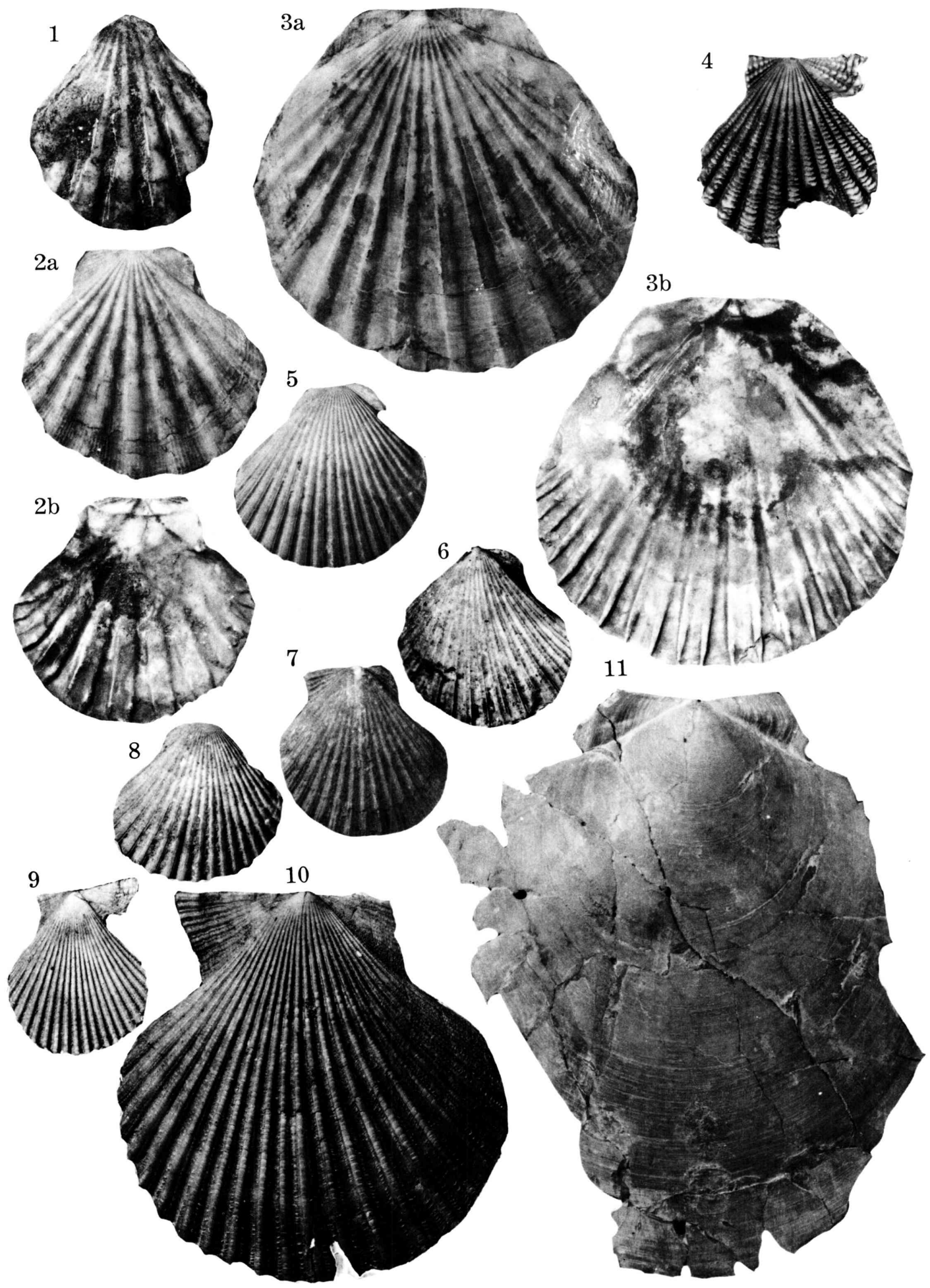

Y. SATo et al.: Pelecypod Fauna of the Shimajiri Group in Miyako-jima 
Dimensions (in $\mathrm{mm}$ ) :-

$\begin{array}{lccccccc}\text { Valve } & \text { Right } & \text { Right } & \text { Right } & \text { Left } & \text { Left } & \text { Left } & \text { Intact v. } \\ \text { Height } & 40.0 & 37.0 & 35.0 & 44.0 & 39.5 & 36.5 & 33.0 \\ \text { Length } & 41.0 & 37.8 & 34.5 & 47.0 & 40.0 & 38.8 & 32.5 \\ \text { Depth } & 11.0 & 10.0 & 10.4 & 15.0 & 11.5 & 10.0 & 20.0\end{array}$

Occurrence:-Loc. 4 (Common); Loc. 5 (Abundant)

Geologic distribution:-Tenno Formation, Shizuoka Prefecture; Koshiba and Yokosuka Formations, Kanagawa Prefecture; Jizodo Formation, Chiba Prefecture.

Recent distribution:-Honshu (Tsugaru Strait as north limit), Shikoku, Kyushu and East China Sea.

Geologic range:-Pliocene to Recent.

Genus Tucetona IREDALE, 1931

Tucetona hanzawai (NomurA and ZiNBo, 1934)

Pl. 1, Figs. 22a-b

1934. Glycymeris hanzawai NomURA and Zinbo. Sci. Rep., Tohoku Imp. Univ., 2nd Ser., vol. 16, no. 2, p. 152, pl. 5, figs. 3a-b.

1961. Tucetona (Tucetonella) hanzawai. HABE, Col. Illust. Shells Japan, p. 112, pl. 50, fig. 4.

1980. Glycymeris (Tucetona) hanzawai. NodA, Sci. Rep. Inst. Geosci., Univ. Tsukuba, Ser. B, vol. 1, p. 80, pl. 2, figs. 14a-b, 15a-b, 16a-b.

Remarks:-Three specimens were examined. This species is characterized by its small, inflated shell with 20 to 25 , smooth, rounded radial ribs which are broader than their interspaces and crenulated inner ventral margin.

Dimensions (in $\mathrm{mm}$ ):-Left valve, height 14.5, length 12.5, depth 4.5; left valve height 14.0 , length 12.0 , depth 1.5 ; right valve, height 11.0 , length 10.0 , depth 3.5.

Occurrence:-Loc. 5 (Few).

Geologic distribution:-Shinzato Formation, Okinawa Prefecture; Ryukyu Limestone, Kagoshima Prefecture.

Recent distribution:-Southern Japan.

Geologic range:-Pliocene to Recent.

\section{Explanation of Plate 2}

(All figures in natural size unless otherwise stated)

Fig. 1. Comptopallium sp. ef. C. izuensis (Nomura and Niro). Loc. 5. ...23

Fig. 2a-b. Annachlamys sp. (n. sp.), Loc. 5. .....................24

Figs. 3a-b. Amussiopecten praesignis (Yokочама). Loc. 5. ............19

Fig. 4. Gloripallium pallium (LinNaEUS). Loc. 5. .................23

Fig. 5, 6, 7, 8. Cryptopecten nux sematensis (OyAMA). $\times 2.5$. Loc. 5. . . .24

Figs. 9, 10. Chlamys (Mimachlamys) satoi (YокоYAMA). Loc. 3. ........21

Fig. 11. Amusium pleuronectes okinawaensis MASUdA, SATo and

Shuto, n. subsp. Loc. 2. ............................... 


\author{
Family Mytilidae \\ Genus Modiolus Lamarck, 1979 \\ Modiolus sp. \\ Pl. 1, Fig. 23
}

Remarks:-One ill preserved intact shell was examined. Shell rather small, inflated; umbones distinctly behind anterior end; surface with fine concentric growth lines; concentric plications rather strong.

Dimensions (in $\mathrm{mm}$ ):-Height 34.0, length 21.0, thickness 19.0.

Occurrence:-Loc. 2 (Rare).

Family Pectinidae

Genus Amusium RöDING, 1798

Amusium pleuronectes okinawaensis MASUdA, SATo and

SHuTo, n. subsp.

Pl. 2, Fig. 11; Pl. 3, Figs. 1, 2

? 1928b. Pecten (Amusium) japonicus (Gmelin). Үoкоуама, Imp. Geol. Surv. Japan, Rep. no. 101, p. 95, pl. 13, fig. 12.

1933. Pecten (Amusium) pleuromectes (Linnaeus). Nomura, Sci. Rep., Tohoku Imp. Univ., 2nd Ser., vol. 16, no. 1, p. 60, pl. 3, fig. 23.

?1958. Amusium japonicum. MakiYAMA, Palaeont. Soc. Japan, Spec. Paper, no. 6 , pl. 99 , fig. 12 .

1981. Amusium pleuronectes. Masuda and Ogasawara, Mem. Vol. Prof. M. OMORI, pl. 2, fig. 5 .

Holotype:-GK-L 11827.

Type locality:-Loc. 2. Oura Formation.

Diagnosis:-Shell generally large and thick. Cardinal and auricular crura large and distinct. Auricular crura terminate distally in a conspicuous oblong denticle. Internal radial ribs stout and its total number 20.

Description:-Shell medium to large in size, medium in thickness, orbicular in outline, compressed, subequivalve, forming an angle of about $120^{\circ}$ at apex. Right valve slightly inflated, smooth, with fine incremental lines; auricles medium in size, nearly equal to each other, a little rounded at anterior and posterior margins, with very shallow byssal notch below anterior auricle and with fine incremental lines; hinge line a little angulated. Left valve slightly inflated, with about 11, very low, faint, fine radial threads near beak and fine incremental lines; radial threads tend to disappear immediately downwards; auricles a little rounded at anterior and posterior margins, with fine incremental lines; hinge line straight. Hinge with conspicuous cardinal crura provided with fine provinculum, wide and shallow resilial pit and distinct auricular crura terminating distally in a conspicuous oblong denticle at each extremity. Interior surface with about 22, distinct internal ribs terminating distally near ventral margin.

Comparison:- The present subspecies is closely related with Amusium pleuronectes (LINNAEUS), a Recent species of Southeast Asia (HABE, 1964), 
and it has hitherto been considered to be a synonym of pleuronectes (NomURA, 1933). However, okinawaensis is obviously distinguished from pleuronectes by its rather large, moderately thick shell, shallow but distinct byssal notch below anterior auricle, distinct, thick internal ribs which are more prominent at lower half of shell and hinge with conspicuous cardianl crura having rather distinct auricular crura terminating distally in a distinct oblong denticle at each extremity. It is treated here as a subspecies of pleuronectes.

Amusium hulshofi (MARTIN, 1909) from the Pliocene of Java, Indonesia, can be distinguished from the present one by its small shell and larger number of internal ribs which terminate at ventral margin. Also this new subspecies is distinguishable from Amusium japonicum (GMELIN), a Recent species of Southern Japan, by its small, rather thick shell, rounded auricles with very shallow byssal notch below anterior auricle and smaller number of internal ribs.

Dimensions (in $\mathrm{mm}$ ) :-

$\begin{array}{lcccccc}\text { Valve } & \text { Right } & \text { Right } & \text { Right } & \text { Left* } & \text { Left } & \text { Left } \\ \text { Height } & 92 & 92 & 69 & 92 & 79 & 49 \\ \text { Length } & 92 & 93.5 & 68 & 90 & 74 & 47 \\ \text { Hinge-Length } & 32 & \text { ca. } 30 & 23 & 34 & 28 & 20 \\ \text { Depth } & 9 & \text { ca. } 10 & 7 & 9 & - & 6\end{array}$

* Holotype

Occurrence:-Loc. 2. (Abundant); Loc. 4. (Abundant).

Geologic distribution:-Byoritsu Formation, Taiwan: ? Canguinsa Formation, Philippines.

Geologic range:-Pliocene.

Genus Amussiopecten SACCO, 1897

Amussiopecten praesignis (Yокочама, 1922)

Pl. 2, Figs. 3a-b

1922. Pecten praesignis Yoкоуама. Jour. Geol. Soc. Japan, vol. 29, no. 350, p. 1, pl. 5, figs. 1-3.

1926. Pecten praesignis. Yokоyama, Jour. Fac. Sci., Imp. Univ. Tokyo, Ser. 2, vol. 1 , pt. 9 , p. 357 , pl. 40 , figs. 1,2 , pl. 41 , fig. 1 .

1931. Amusium (Amussiopecten) praesignis. KURodA, Venus vol. 3, apped., p. 77 , fig. 80.

1932. Amussiopecten praesignis. MakiYama, Iwanami-koza, pl. 1, fig. 9.

1933. Amusium (Amussiopecten) praesignis. Kuroda, Ibid., p. 55, fig. 35.

1938. Amussiopecten praesignis. OTUKA, Jour. Fac. Sci., Imp. Univ. Tokyo, Sec. 2, vol. 5, pts. 1 \& 2, p. 6, pl. 1, fig. 2.

1941. Pecten praesignis. YABE and HATAI, Jour. Geol. Soc. Japan, vol. 48, no. 576 , p. 464 , pl. 11 , fig. 1.

1955. Amussiopecten praesignis. Shuto, Trans. Proc. Palaeont. Soc. Japan, N. S., no. 20 , p. 103 , pl. 17 , figs. $1,3$.

1957. Amussiopecten praesignis. Akryama, Ibid., no. 25, p. 33, pl. 7, figs. 5, 6 .

1958. Amussiopecten praesignis. MakiYama, Palaeont. Soc. Japan, Spec. Paper, no. 4 , pl. 52, figs. 1,2 , pl. 53, fig. 1 .

1960. "Pecten" sp. AoKI, Trans. Proc. Palaeont. Soc. Japan, N. S., no. 39, pl. 34, figs. 7-9. 
1962. Amussiopecten praesignis. MasudA, Sci. Rep., Tohoku Univ., 2nd Ser., vol. 33, no. 2, p. 226, pl. 27, figs. 4, 5 .

1966. Amussiopecten praesignis. AoKI, Trans. Proc. Palaeont. Soc. Japan, N. S., no. 62 , p. 255 , pl. 31 , figs. $8 \mathrm{a}-\mathrm{b}$.

1973. Amussiopecten praesignis. HAYASAKA, Sci. Rep., Tohoku Univ., 2nd Ser., Spec. Vol., no. 6, p. 101, pl. 6, fig. 2.

1973. Amussiopecten praesignis. KanNo and ChaNG, Geol. Palaeont. Southeast Asia, vol. 12, pl. 30, fig. 11.

1978. Amussiopecten praesignis. Окамото and HonzA, Jour. Geol. Soc. Japan, vol. 84 , no. 10 , fig. 3 .

1978. Amussiopecten praesignis. Окамото, Geol. Surv. Japan, Cruse Rep., no. 10, figs. X-I, 1a-b.

1980. Amussiopecten sp. NodA, Sci. Rep. Inst. Geosci., Univ. Tsukuba, Ser. B, vol. 1 , p. 83 , pl. 4 , fig. 17 .

1980. Amussiopecten praesignis. MASUdA, Saito Ho-on Kai Mus. Nat. Hist., Res. Bull., no. 48, pl. 3, fig. 2.

1981. Amussiopecten praesignis. Masuda and Ogasawara, Mem. Vol. Prof. $M$. OMORI, pl. 2, fig. 4.

1981. Amussiopecten praesignis. TsuchI (ed.), Plates of Selected Index Fossils. Neogene of Japan, pl. 4, fig. 2.

Remarks:-The present species is characterized by the following features:Shell large, medium in thickness, compressed, somewhat longer than high, equilateral except for auricles, inequivalve, right valve more convex than left valve; both valves radiately ribbed and forming an angle of about $110^{\circ}$ at apex. Right valve moderately inflated, with 15 to 19 , low, broad, more or less squarish, roundly flat-topped radial ribs and distinct, lamellated fine concentric growth lines on back of radial ribs and interspaces between radial ribs; radial ribs broader than their interspaces, tend to become gradually obsolete towards ventral and lateral margins, sometimes with faint, fine longitudinal striae near ventral and lateral margins but recognized by reflected light only; radial ribs near submargins much lower than those of central part of disc, tend to divide into two parts towards ventral margin; interspaces between radial ribs sometimes with faint, fine longitudinal striae recognizable by reflected light; hinge line forms an angle of about $160^{\circ}$ at beak; auricles medium in size, subequal to each other, with rather distinct, lamellated, fine concentric lines and very faint, fine radial threads; anterior with wide and shallow byssal notch, somewhat folded near submargin; hinge with prominent cardinal crura with fine provinculum, wide and deep resilial pit provided with faint, fine lateral ridge, rather distinct auricular crura which extend along inner margins of auricles and terminate distally in a rounded, more or less oblong denticle at each extremity of hinge. Left valve nearly flat in younger stage but tends to become slightly inflated with growth, with low, round-topped radial ribs and distinct, lamellated, fine concentric growth lines; radial ribs narrower than their interspaces but sometimes nearly equal to their interspaces in breadth at ventral margin; radial ribs near submargins slender, very low, with faint, fine intercalary threads; submargins somewhat more elevated than disc surface in younger stage; auricles subequal, sculptured with rather distinct lamellated concentric lines; hinge line straight; hinge with auricular crura which ter- 
minate distally in a rounded denticle at each extremity. Interior surface with prominent paired internal ribs running from upper part of disc to ventral margin.

Comparison:-The present species is closely related with Amussiopecten iitomiensis (OTUKA) described from the Miocene Shizukawa Formation in Yamanashi Prefecture, but iitomiensis can be distinguished from the present one by its rather smaller number of radial ribs which do not usually bifurcate and narrower interspaces compared with those of praesignis. Also Amussiopecten singkirensis (MARTIN, 1903) described from the Pliocene of Java, Indonesia, can be distinguished from the present one by its rounded radial ribs on right valve and roof-shaped radial ribs on left valve.

O'HARA and ITO (1980) described the present species from the Miocene Senhata Formation, Chiba Prefecture, but their specimens differ from praesignis in having rounded radial ribs.

Dimensions (in $\mathrm{mm}$ ) :-

$\begin{array}{lccccccc}\text { Valve } & \text { Right* } & \text { Right* } & \text { Left* } & \text { Left* } & \text { Right } & \text { Righ } & \text { Left } \\ \text { Height } & 62.0 & 40.0 & 86 & 83 & 45.7 & 36 & 58.5 \\ \text { Length } & 63.5 & 41 & 95 & 89 & 48 & 38 & 60 \\ \text { Depth } & 8 & 4 & 9 & - & 9 & 8 & 7.5\end{array}$

* Specimens from Nakoshi Formation, Okinawa-jima

Occurrence:-Loc. 5 (Abundant).

Geologic distribution:-Dainichi, Hamaishidake and Shirahama Formations, Shizuoka Prefecture; Kurotaki Formation, Chiba Prefecture; Ananai and Koe Formations, Kochi Prefecture; Takanabe Member of Koyu Formation, Miyazaki Prefecture; Nakoshi, Shinzato and Maja Formations, Okinawa Prefecture; Nakazato Formation, Kanagawa Prefecture; Tajima Formation, Kagoshima Prefecture; Off Mishima Island, Yamaguchi Prefecture; Byoritsu Formation, Taiwan.

Geologic range:-Pliocene.

Genus Chlamys (Bolten) RöDING, 1978

Subgenus Mimachlamys IREDALE, 1929

Chlamys (Mimachlamys) satoi (YoкоYama, 1928)

Pl. 2, Fig. 9, 10

1928b. Pecten (Chlamys) satoi Yoкочамa. Imp. Geol. Surv. Japan, Rep., no. 101 , p. 94 , pl. 13 , figs. 13,14 , pl. 14, fig. 2.

1932. Pecten kaisakiensis Nomura and NinNo. Sci. Rep., Tohoku Imp. Univ., 2nd Ser., vol. 15, no. 3, p. 177, pl. 12, figs. 3, 4.

1960. Gloripallium satowi (Yокоуама). Sнuтo, Mem. Fac. Sci., Kyushu Univ., Ser. $D$, vol. 9 , no. 3 , p. 119 , pl. 12 , fig. 5 .

1960. Mimachlamys cf. miniacea (LAMARCK). ShUto, Ibid., vol. 9, no. 3, p. 122, pl. 13, fig. 4.

1960. Chlamys satoi. MakiYama, Palaeont. Soc. Japan, Spec. Paper, no. 6, pl. 99 , figs. 13, 14, pl. 100, fig. 2.

1962. Chlamys (Mimachlamys) satoi. MasUdA, Sci. Rep., Tohoku Univ., 2nd Ser., vol. 33 , no. 2 , p. 189 , pl. 19 , figs. 18, 19, pl. 22 , fig. 14. 
1980. Chlamys satoi. Masuda, Saito Ho-on Kai Mus. Nat. Hist., Res. Bull., no. 48 , pl. 3 , fig. 1 .

1981. Chlamys satoi. Masuda and Ogasawara. Mem. Vol., Prof. M.OMori, pl. 2, fig. 3 .

Description of the Miyako specimens:-Shell medium in thickness, higher than long, equilateral except for auricles, inequivalve; left valve more convex than right valve; valves radiately ribbed and forming an angle of a little less than $90^{\circ}$ at apex. Right valve slightly inflated, with 25 to 30 , distinct, more or less squarish, scaled radial ribs, rather distinct fine concentric growth lines; radial ribs broader than their interspaces, accompanied with imbricated, fine radial threads on both sides, with regularly spaced, more or less lamellated concentric lines; interspaces between radial ribs with fine, somewhat lamellated concentric lines, sometimes with scaled, fine intercalary threads in full adult specimens; scales thin, inclined apically, regularly spaced; auricles medium in size, anterior one larger than posterior one, with deep and wide byssal notch and wide byssal area, with several, finely scaled radial threads and distinct, somewhat lamellated concentric lines; hinge with distinct but simple cardinal crura, wide and shallow resilial pit with short, widely opening lateral ridges, anterior one much shorter than other, and conspicuous ctenolium.

Left valve moderately inflated, with distinct, more or less squarish, imbricated radial ribs accompanied with a imbricated, fine radial threads on both sides of radial ribs towards ventral margin, imbricated fine intercalary threads and distinct fine concentric lines of growth; radial ribs nearly equal to their interspaces in breadth, flatly round-topped at upper part of disc, but tend to become distinctly imbricated ventrally; interspaces between radial ribs with a single or sometimes two, imbricated, fine intercalary threads which usually appear at about middle part of disc, with distinctly lamellate concentric lines; anterior auricle larger than posterior one with numerous, finely imbricated radial threads and distinct concentric lines; posterior auricle similar to anterior in sculpture. Interior surface gently folded corresponding to radial ribs and with marginal serration.

Comparison:-The present species can be distinguished from Chlamys (Mimachlamys) nobilis (REEVE), a Recent species of Southern Japan, by its larger number of radials which are accompanied by a imbricated radial thread on both sides and inequivalved shell. Pecten kakisakiensis described from the Shirahama Formation, Shizuoka Prefecture (NomURA and NiINo, 1932), is a synonym of the present species, because the surface sculpture of both are quite similar to each other and the number of radial ribs of kakisakiensis varies from 25 to 30 .

\begin{tabular}{|c|c|c|c|c|c|c|}
\hline Valve & Right & Right & Right & Left & Left & Left \\
\hline Height & 38.0 & 28.0 & 26.0 & 56.6 & 39.5 & 30.5 \\
\hline Length & 34.0 & 25.0 & 22.5 & 58.0 & 34.0 & 27. \\
\hline Depth & 5.0 & 3.5 & 3.2 & 10.0 & 8.0 & \\
\hline
\end{tabular}


Geologic distribution:-Byoritsu Formation, Taiwan; Nakoshi and Maja Formations, Okinawa Prefecture; Tajima Formation, Kagoshima Prefecture; Takanabe Member of Koyu Formation, Miyazaki Prefecture; Ananai Formation, Kochi Prefecture; Dainichi and Shirahama Formations, Shizuoka Prefecture.

Geologic range:-Pliocene.

Genus Comptopallium IREDALE, 1939

Comptopallium sp. cf. C. izuensis (Nomura and NinNo, 1932)

Pl. 2, Fig. 1

\title{
Compared with:-
}

1932. Pecten izuensis Nomura and Nino. Sci. Rep., Tohoku Imp. Univ. 2nd Ser., vol. 15, no. 3, p. 179, pl. 11, figs. 9-13.

Remarks:-One water-worn specimen was examined. Shell rather small, higher than long, moderately inflated, with seven, distinct round-topped radial ribs which are a little broader than their interspaces, and subordinate radial ribs at submargins, but any fine structures are not preserved on radial ribs and in their interspaces.

Dimensions (in $\mathrm{mm}$ ) :-Right valve, height 33.0, length 29.0, depth 7.4. Occurrence:-Loc. 5 (Rare).

\author{
Genus Gloripallium IREDALE, 1939 \\ Gloripallium pallium (LINNAEUS, 1758) \\ Pl. 2, Fig. 4
}

1842. Pecten pallium (Linnaeus). Sowerby, Thes. Conch., vol. 1, p. 73, pl. 17, figs. 148-150, pl. 18, figs. 167, 168.

1853. Pecten pallium. ReEve, Conch. Icon., vol. 8, Pecten, sp. p. 63, pl. 17, figs. 63a-c.

1888. Pecten pallium. Küster und Kobelt in Martini und Chemnitz, Syst. Conch. Cab., vol. 7, p. 39, p. 103, pl. 11, figs. 1, 5, pl. 28, figs. 7, 8.

1941. Pecten pallium. HataI, Troop. Indust. Inst., Bull., no. 7, p. 59, pl. 57, figs. 1, 2, 6, 7 .

1951. Gloripallium pallium. HABE, Genera Japan. Shells, no. 1, p. 76, fig. 152. 1977. Gloripallium pallium. HABE, Syst. Moll. Japan, p. 84, pl. 17, fig. 5.

Remarks:-One well preserved small right valve and two fragmental specimens were examined. The specimens are characterized by its rather thick shall with about 12 , elevated, conspicuously and imbricately scaled radial ribs which are divided into two or three parts by shallow longitudinal furrows towards ventral margin, a few, fine, scaled intercalary threads, auricles with a few nodulose radial threads, distinct ctenolium and fine marginal serration in interior surface, and they are reasonably identical to Gloripallium pallium (LINNAEUS).

The present record may be the first from the geological formation. 
Dimensions (in mm) :-Right valve, height 30.2, length 27.5, depth ca. 6.0. Occurrence:-Loc. 5 (Few).

Recent distribution:-Southeast Asia to Southern Japan (Kii Peninsula as north limit).

Geologic range:-Pliocene to Recent.

Genus Annachlamys IREDALE, 1939

Annachlamys sp.

Pl. 2, Figs. 2a-b

Remarks:-One left valve was examined. This is characterized as follows: -Shell rather small, rather thick, compressed, forming an angle of about $100^{\circ}$ at apex. Valve nearly flat in younger stage but tends to become a little inflated with growth, with nine, round-topped radial ribs and subordinate radial ribs at submargins; radial ribs superimposed with several, fine radial threads which appear at upper part of disc; radial ribs somewhat broader than their interspaces having fine radial threads and densely lamellate, fine concentric lines. Hinge with distinct cardinal crura ornamented by fine but rather distinct provinculum and distinct auricular crura terminating distally distinct denticle at each extremity. Interior surface with distinct, paired internal ribs which are more prominent near ventral margin.

The present specimen can be distinguished from Annachlamys reevi (ADAMS and REEVE), a Recent species of Southern Japan, by its smaller number of radial ribs without scales.

It seems to represent an unnamed species but naming is withheld until more specimens are collected. Anachlamys is a rare genus in the Cenozoic formations of Japan and the present record may be the first.

Dimensions (in $\mathrm{mm}$ ) :-Left valve, height 36.2 , length 38.4 , depth 6.0.

Occurrence:-Loc. 5 (Rare).

Genus Cryptopecten DALL, BARTSCH and REHDER, 1938

Cryptopecten nux sematensis (OYAMA, 1973)

Pl. 2, Figs. 5, 6, 7, 8

1922. Pecten tissotii Bernardi. Yokoyama, Jour. Coll. Sci., Imp. Univ. Tokyo, vol. 44, art. 1, p. 182, pl. 15, figs. 1, 2 (non BerNARdi, 1958).

1954. Aequipecten (Cryptopecten) sematensis OYAMA. In TAKI and OYAMA, Palaeont. Soc. Japan, Spec. Paper, No. 2, pl. 35, figs. 1, 2.

1973. Aequipecten (Cryptopecten) sematensis. OYAMA, Ibid., no. 7, p. 85, pl. 34, figs. 9, 10.

1981. Aequipecten sp. NodA, Sci. Rep. Inst. Geosci., Univ. Tsukuba, Sec. B, vol. 1 , p. 82 , pl. 12 , fig. 21 .

Remarks:-The present subspecies, first illustrated as Pecten tissotii (BERNARd) by YoKoyama (1922) from the Pleistocene Semata Formation in Chiba Prefecture, was considered to represent an undescribed species by 
OYAMA (1954). The present writers give a redefinition of OYAMA's sematensis with designation of the type specimen.

The material in hand is characterized by the following features:- Shell small, moderatelly thick, suborbicular; subequivalve, right valve being a little more inflated than left valve, inequilateral, length of posterior side being longer than that of anterior side, forming an angle of about $90^{\circ}$ at apex. Right valve with about 20 , more or less squarish, rounded radial ribs accompanied with a fine radial thread on both sides of radial ribs, several, fine imbricated radial threads in interspaces between radial ribs; anterior auricle with fine radial threads and concentric lines, and furnished with rather deep byssal notch. Hinge with a ctenolium and conspicuous cardinal crura ornamented by fine provinculum. Interior surface smooth except for distinct marginal serration. Left valve with sculpture similar to right valve. They are radically identified with "Cryptopecten sematensis OYama."

C. sematensis is so closely related with C. nux (REEVE, 1853), a Recent species of Southeastern Pacific, that they can hardly be distinguished from each other at species level. However, they reveal a slight but clear difference, that is, the latter has inflated and equivalve shell. Under this circumstance, $C$. sematensis is treated here as a subspecies of $C$. nux. Also the present one can be distinguished from Cryptopecten vesiculosus (DUNKER, 1877), a common Recent species of Southern Japan, by its smaller shell, inflated left valve and larger number of radial ribs.

Dimensions (in $\mathrm{mn}$ ) :-

$\begin{array}{lcrrrr}\text { Valve } & \text { Right } & \text { Right } & \text { Left } & \text { Left } & \text { Left } \\ \text { Height } & 16.5 & 13.0 & 15.4 & 14.8 & 14.3 \\ \text { Length } & 17.0 & 12.0 & 15.0 & 14.5 & 14.8 \\ \text { Depth } & 4.8 & 4.0 & 3.5 & 3.2 & 3.0\end{array}$

Occurrence:-Loc. 5 (Common).

Geologic distribution:-Semata Formation, Chiba Prefecture; Shinzato Formation, Okinawa Prefecture.

Geologic range:-Pliocene to Pleistocene.

\author{
Family Plicatulidae \\ Genus Plicatula LAMARCK, 1801 \\ Plicatula sp. \\ P1. 3, Figs. 4a-b
}

Remarks:-Two water-worn specimens were examined. The present specimens are characterized by their small shell, distinctly elevated cardinal teeth, and wide and deep resilifer pit between anterior and posterior teeth and are referred to Plicatula LAMARCK, 1801. Further comparison is difficult because of imperfect preservation.

Dimensions (in $\mathrm{mm}$ ):-Height 20.0, length 16.0, depth 5.5; height 8.5, length 6.0, depth 1.5.

Occurrence:-Loc. 5 (Rare). 


\author{
Family Anomiidae \\ Genus Anomia Linnaeus, 1758 \\ Anomia chinensis PHILlIPPI, 1849 \\ Pl. 3, Figs. 3a-b
}

1859. Anomia cytaeum Gray. ReEve, Conch. Icon., vol. 11, Anomia, sp. 10, pl. 2, figs. 10a-b.

1868. Anomia chinensis PhilipPI. In MARTINi und Chemnitz, Syst. Conch. Cab., vol. 7, pt. 1, p. 58, pl. 6, figs. 1, 2.

1920. Anomia cytaeum Gray, var. Yokoyama, Jour. Coll. Sci., Imp. Univ. Tokyo, vol. 39 , art. 6 , p. 146, pl. 11, figs. $17 \mathrm{a}-\mathrm{b}$.

1920. Anomia nipponensis Yokoyama. Ibid., vol. 39, art. 6, p. 146, pl. 11, figs. 18a-b.

1928. Anomia lischkei DaUtzenberg and Fischer. Yokoyama, Imp. Geol. Surv. Japan, Rep., no. 101, p. 91, pl. 14, figs. 4, 5.

1930. Anomia lischkei. Matsumoto, Sci. Rep., Tohoku Imp. Univ., 2nd Ser., vol. 13, no. 3 , p. 107 , pl. 39 , fig. 12 .

1931. Anomia lischkei. Kuroda in Honma, Geol. Cent. Shinano, p. 40, pl. 3, figs. 14, 15.

1932. Anomia lischkei. Kuroda, Venus, vol. 3, no. 4, append., p. 119, fig. 121.

1932. Anomia cytaeum. Kuroda, Ibid., vol. 3, no. 4, append., p. 120, fig. 122.

1935. Anomia cytaeum. Nomura and HataI, Saito Ho-on Kai Mus., Res. Bull., no. 6 , p. 107 , pl. 11 , figs. 5,6 .

1938. Anomia cytaeum. Nomura, Sci. Rep., Tohoku Imp. Univ., 2nd Ser., vol. 19, no. 2, p. 251, pl. 35, fig. 7 .

1953. Anomia chinensis. HABE in KurodA, Illust. Cat. Japan. Shells, no. 24, p. 198, pl. 27, fig. 12 , pl. 28, fig. 21 .

1954. Anomia lischkei. Ozaki, Fukuta and Ando, Bull. Nat. Sci. Mus., vol. 3, no. 3, p. 169 , pl. 29 , figs. $19 \mathrm{a}-\mathrm{b}$.

1954. Anomia lischkei. TAKI and OYAMA, Palaeont. Soc. Japan, Spec. Paper, no. 2, pl. 12, figs. $17 \mathrm{a}-\mathrm{b}$.

1958. Anomia sinensis PhilipPi. Yамамото and HABe, Bull. Mar. Biol. Sta. Asamushi, Tohoku Univ., vol. 9, no. 1, p. 17, pl. 2, figs. 11, 21.

1958. Anomia lischkei. Ozaki, Bull. Nat. Sci. Mus., N. S., vol. 4, no. 1, p. 119, pl. 24, fig. 5 .

1958. Anomia cf. lischkei. FuJIE, Cen. Res., no. 28, p. 670, pl. 28, fig. 25.

1960. Anomia sinensis. HABE, Publ. Shirikishinai Mar. Sta. Biol. Instruct., no. 2 , pl. 4 , figs. 4,7 .

1961. Anomia chinensis. Hayasaka, Sci. Rep., Tohoku Univ., 2nd Ser., vol. 33, no. 1 , p. 33 , pl. 3, fig. 7 .

1962. Anomia lischkei. Kamada, Palaeont. Soc. Japan, Spec. Paper, no. 8, p. 71, pl. 3, figs. 8-11.

\title{
Explanation of Plate 3
}

(Natural size)

Figs. 1, 2. Amusium pleuronectes okinawaensis MASUdA, SATo and

Shuto, n. subsp. Holotype, Reg. No. GK-L 11827. Loc. 2. . . . . . . . .18

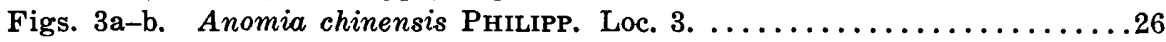

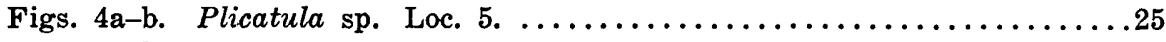

Figs. 5a-b. Ostrea denselamellosa LischKe. Loc. $2 . \ldots \ldots \ldots \ldots \ldots \ldots \ldots \ldots$

Fig. 6. Crassostrea gigas (ThunBERG). Loc. 1. . . . . . . . . . . . . . . . 29 


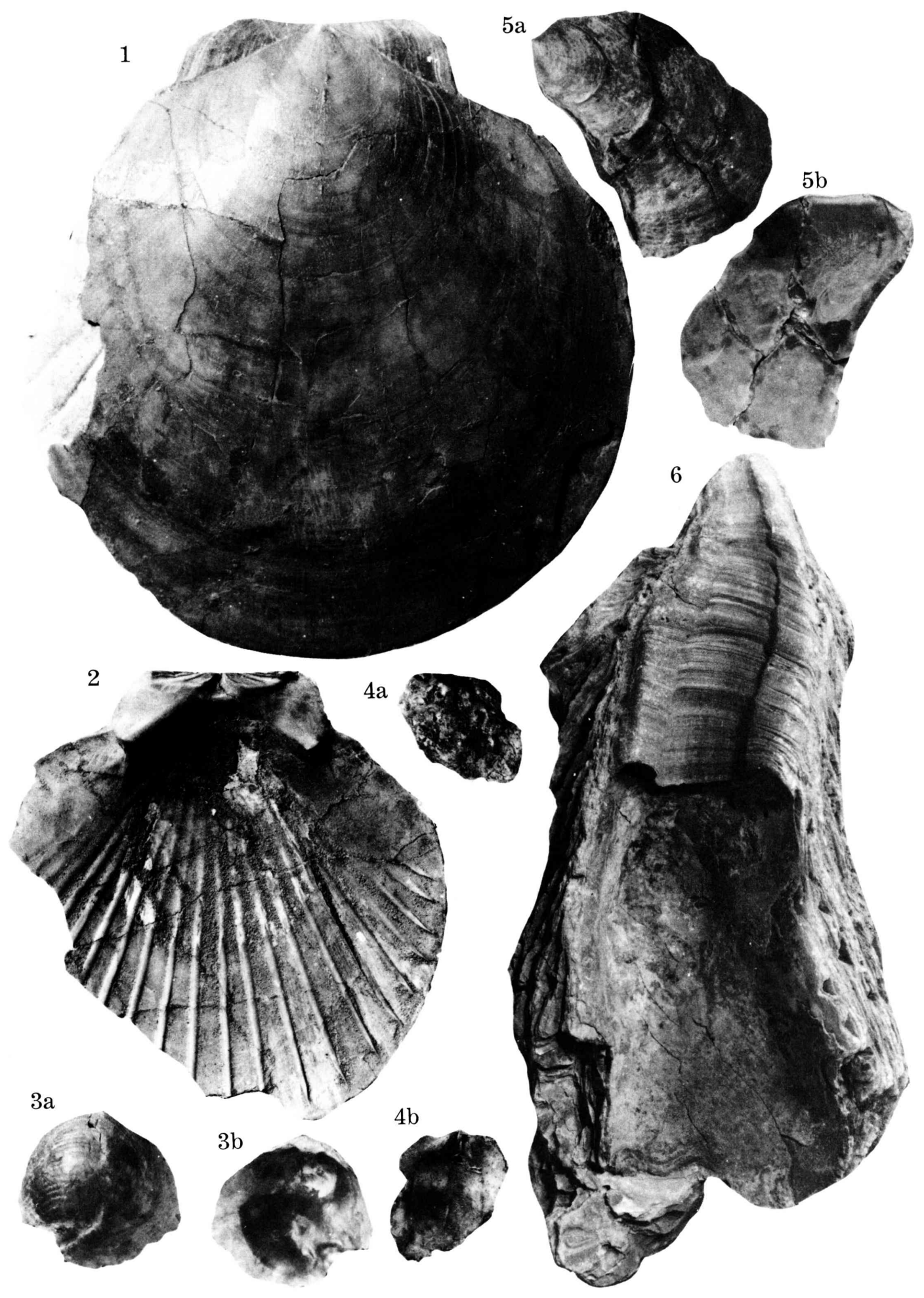

Y. SAto et al.: Pelecypod Fauna of the Shimajiri Group in Miyako-jima 


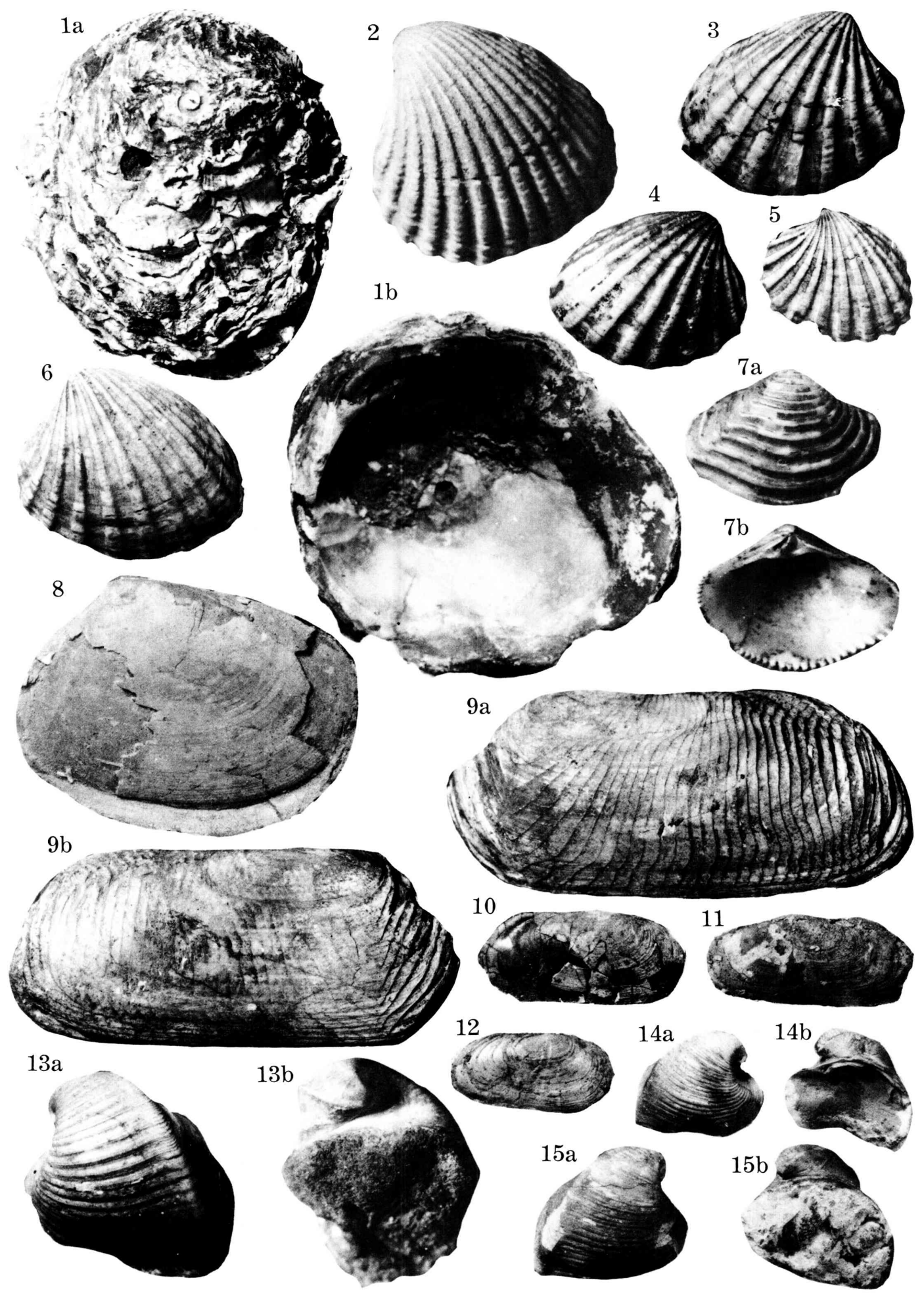

Y. SATo et al.: Pelecypod Fauna of the Shimajiri Group in Miyako-jima 
1965. Anomia cytaeum. KASENo and MAtsuUra, Sci. Rep., Kanazawa Univ., vol. 10 , no. 1 , pl. 8 , fig. 7 .

1967. Anomia lischkei. HAse, Contr. Inst. Geol. Paleont., Tohoku Univ., no. 64, pl. 2, figs. 3, 3a.

1969. Anomia chinensis. Hayasaka, Rep. Fac. Sci., Kagoshima Univ., no. 2, p. 42 , pl. 2, figs. 3a-b.

1969. Anomia chinensis Matsushima, Bull. Kanagawa Pref. Mus., vol. 1, no. 2, pl. 10, fig. 4 .

1971. Anomia chinensis. Kuroda, Habe and OYama, Sea Shells Sagami Bay, p. 590, pl. 84, figs. 12-14.

1973. Anomia cytaeum. OYAMA, Palaeont., Soc. Japan, Spec. Paper, no. 17, p. 88 , pl. 28, figs. 2, 3, 6 .

1974. Anomia chinensis. Shibata in Itoigawa, Shibata and Nishimoto, Bull. Mizunami Foss. Mus., no. 1, p. 71, pl. 15, figs. 5-7b.

1977. Anomia chinensis. HABE, Syst. Moll. Japan, p. 97, pl. 18, figs. 2, 3, 6.

Remarks:-The specimens hitherto known as cytaeum, lishkei and nipponensis from the Japanese waters and younger Cenozoic strata of Japan were considered to be a synonym of chinensis (HABE, 1953, 1977; KURODA, HABE and OYAMA, 1973, etc.) and the writers agree with this classification.

The shape of shell in the present species is variable from suborbicular to fan-shaped and also surface is sculptured by radial striation or sometimes almost smooth.

Dimensions (in $\mathrm{mm}$ ):-

$\begin{array}{lrrrrr}\text { Valve } & \text { Left } & \text { Left } & \text { Left } & \text { Left } & \text { Left } \\ \text { Height } & 36.0 & 25.0 & 24.5 & 24.0 & 20.0 \\ \text { Length } & 33.5 & 26.0 & 23.0 & 22.0 & 19.0 \\ \text { Depth } & 5.0 & - & 6.0 & - & 3.5\end{array}$

Occurrence:-Loc. 2 (Common); Loc. 3 (Common).

Geologic distribution:-Moniwa, Tasunokuchi and Nakatsuyama Formations, Miyagi Prefecture; Yamanouchi Formation, Gifu Prefecture; Shigarami Formation, Nagano Prefecture; Omma Formation, Ishikawa Prefecture; Honya Formation, Fukushima Prefecture; Koshiba, Naganuma and Sakuragicho Formations, Kanagawa Prefecture; Toshima Formation, Aichi Prefecture; Katori Formation, Chiba Prefecture; Daishaka Formation, Aomori Prefecture; Kawa-

\section{Explanation of Plate 4}

(All figures in natural size unless otherwise stated)

Fig. 1a-b. Chama japonica LAmARCK. Loc. 5. ......................

Fig. 2. Cyclocardia sp. cf. C. ferruginea (ClESSIN). $\times 3.5$. Loc. $3 . \ldots \ldots . .30$

Figs. 3, 4, 5, 6. Megacardita granulicostata (NomURA). $\times 1.5$. Loc. 5. ...31

Figs. 7a-b. Bathytormus foveolatus (SOWERBY). $\times 3.5$. Loc. $5, \ldots \ldots \ldots 31$

Fig. 8. Psammotreta (Pseudometis) praerupta (SALISBURY). Loc. 2. ......33

Figs. 9a-b. Solecurtus miyakojimaensis MASUdA, SATo and ShUto, n. sp.

Holotype, Reg. No. GK-L 11828. Loc. 2. ..................... 33

Figs. 10, 11, 12. Azorinus abbreviatus (GouLD). Loc. 2. .............. 34

Figs. 13a-b, 14a-b, 15a-b. Meiocardia vulgaris (REEVE), 13a-b, $\times 3.5$.

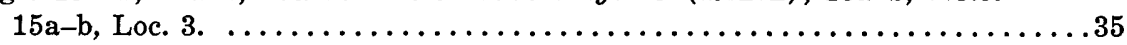


chi Formation, Kagoshima Prefecture; Byoritsu Formation, Taiwan. Recent distribution:-Southern Hokkaido, Honshu, Shikoku, Kyushu, Korea, China and Southeast Asia.

Geologic range:-Miocene to Recent.

\author{
Family Ostreidae \\ Genus Ostrea LinNaEus, 1758 \\ Ostrea denselamellosa LISCHKE, 1869
}

Pl. 3, Figs. 5a-b

1869. Ostrea denselamellosa LischKe. Japan. Meer. Conchy., vol. 1, p. 177, pl. 13, figs. a, b, pl. 14, fig. 1 .

1906. Ostrea denselamellosa. TokUnAgA, Jour. Coll. Sci., Imp. Univ. Tokyo, vol. 21 , art. 2 , p. 68 , pl. 4 , fig. 6 .

1920. Ostrea gigas ThUNBerG. YokoYama, Ibid., vol. 39, art. 6, p. 162, pl. 15, figs. $1,2$.

1926. Ostrea denselamellosa. Yokoунмa, Jour. Fac. Sci., Imp. Univ. Tokyo, Ser. 2, vol. 1, pt. 9, p. 375, pl. 43, fig. 11.

1928. Ostrea denselamellosa. YoкоYамA, Imp. Geol. Surv. Japan, Rep., no. 101, p. 100, pl. 16, fig. 1 .

1929. Ostrea denselamellosa. WAKIYA, Japan. Jour. Zool., vol. 22, no. 3, p. 366, pl. 9, figs. 3, 4 .

1930. Ostrea denselamellosa. KURodA, Venus, vol. 2, append., p. 49, fig. 55.

1933. Ostrea denselamellosa. Nomura, Sci. Rep., Tohoku Imp. Univ., 2nd Ser., vol. 16 , no. 1 , p. 46 , pl. 4 , fig. 6 .

1954. Ostrea denselamellosa. TAKI and OYAMA, Palaeont. Soc. Japan, Spec. Paper, no. 2, pl. 16, figs. 1, 2.

1961. Ostrea (Ostrea) denselamellosa. HAYASAKA, Sci. Rep., Tohoku Univ., 2nd Ser., vol. 33 , no. 1 , p. 34 .

1963. Ostrea denselamellosa. YAMAdA, Bull., Fac. Art. Sci., Mie Univ., no. 43, figs. $5 a-b$.

1971. Ostrea denselamellosa. NodA, Trans. Proc. Palaeont. Soc. Japan, N. S., no. 81, p. 40, pl. 7 , figs. 10,18 .

1971. Ostrea denselamellosa. KURODA, HABE and Oyama, Sea Shells Sagami Bay, p. 595, pl. 86, figs. 2, 3.

1973. Ostrea denselamellosa. Oyama, Palaeont. Soc. Japan, Spec. Paper, no. 17, p. 89, pl. 30, figs. 1a-b.

1977. Ostrea denselamellosa. HABE, Syst. Moll. Japan, p. 110, pl. 20, fig. 5.

Remarks:-Several water worn specimens were examined. The present specimens are characterized by their rather thick shell with radial ribs and concentric lines, and small crenulations along anterior and posterior margins at ligamental area and are reasonably identified to Ostrea denselamellosa LISHCHKE.

Occurrence:-Loc. 2 (Few); Loc. 3 (Few); Loc. 5 (Few).

Geologic distribution:-Byoritsu Formation, Taiwan; Haneji Formation, Okinawa Prefecture; Sakishima Formation, Mie Prefecture; Toshima Formation, Aichi Prefecture; Yokosuka Formation, Kanagawa Prefecture.

Recent distribution:-Honshu (Boso Peninsula as north limit), Shikoku, Kyushu and East China Sea.

Geologic range:-Pliocene to Recent. 


\section{Genus Crassostrea SACCO, 1897}

Crassostrea gigas (THUNBERG, 1793)

Pl. 3, Fig. 6

1906. Ostrea gigas, ThUNBERG. Jour. Coll. Sci., Imp. Univ. Tokyo, vol. 21, art. 2 , p. 68 , pl. 4 , figs. $5 a-b$.

1920. Ostrea gigas. YoKoYAMA, Ibid., vol. 39 , art. 6 , p. 162, pl. 15, figs. $3,4$.

1920. Ostrea denselamellosa LischKe. YокоYAMA, Ibid., vol. 39, art. 6, p. 162, pl. 16, figs. $6 \mathrm{a}-\mathrm{b}$.

1920. Ostrea plicata Chemnitz. Yoxoyama, Ibid., vol. 39, art. 6, p. 163, pl. 17, fig. 2.

1928b. Ostrea gigas. Yoкоуама, Imp. Geol. Surv. Japan, Rep., no. 101, p. 99, pl. 15, figs. 2 , 3 .

1931. Ostrea (Crassostrea) gigas. KURODA, Venus, vol. 2, no. 5, append., p. 55, fig. 56.

1931. Ostrea (Crassostrea) gigas. KURodA, in Honma, Geol. Cent. Shinano, p. 32 , pl. 1 , figs. 4,5 .

1934. Ostrea (Crassostrea) gigas. gigas. Kinoshita and ISAHAYA, Hokkaido Fish. Exp. Sta., Rep. Fish Surv., no. 33, p. 14, pl. 10, fig. 73.

1937. Ostrea (Crassostrea) gigas. Nomura and HATAI, Saito Ho-on Kai Mus., Res. Bull., no. 13, p. 126, pl. 18, fig. 3.

1954. Ostrea (Crassostrea) gigas. TAKI and OYAMA, Palaeont. Soc. Japan, Spec. Paper, no. 2, pl. 16, figs. 3, 4, pl. 17, figs. 6a-b.

1954. Ostrea (Crassostrea) gigas. OzAKI, FuKuTA and Ando, Nat. Sci. and Mus., vol. 18 , nos. 5-6, p. 170 , pl. 30 , fig. 27 .

1957. Ostrea (Crassostrea) gigas. OZAKI, Ibid., vol. 24, nos. 3-4, text-fig. 6-1.

1958. Ostrea (Crassostrea) gigas. OzAKI, Bull. Nat. Sci. Mus., N. S., vol. 4, no. 1, p. 118 , pl. 21 , fig. 7 .

1958. Ostrea (Crassostrea) gigas. FuJIE, Cen. Res., no. 28, p. 670, pl. 27, figs. 22, 23.

1959. Ostrea (Crassostrea) gigas. Araki, Trans. Proc. Palaeont. Soc. Japan, N. S., no. 33 , p. 21 , pl. 4 , figs. 1a-b.

1960. Ostrea (Crassostrea) gigas. ARAKI, Bull. Lib. Arts Dep., Mie Univ., Spec. Vol., no. 1 , p. 82, pl. 11, figs. 1a-b.

1960. Ostrea (Crassostrea) gigas. HAYASAKA, Sci. Rep., Tohoku Univ., 2nd Ser., Spec. Vol., no. 4, p. 4, p. 364, pl. 37, fig. 6, pl. 38, figs. $1,2$.

1961. Ostrea (Crassostrea) gigas. HAYASAKA, Sci. Rep., Tohoku Univ., and Ser., vol. 33 , no. 1 , p. 34 .

1965. Ostrea (Crassostrea) gigas. KASENo and MATSUURA, Sci. Rep., Kanazawa Univ., vol. 10, no. 1, pl. 12, fig. 2.

1967. Ostrea (Crassostrea) gigas. HASE, Contr. Inst. Geol. Paleont., Tohoku Univ., no. 64, pl. 3, figs. 9-16.

1967. Ostrea gigas. IwaI and Shiobara, Bull. Educ. Fac., Hirosaki Univ., no. 20, pl. 1, figs. 14a-b, pl. 2, fig. 1 .

1969. Crassostrea gigas. Matsushima, Bull. Kanagawa Pref. Mus., vol. 1, no. 1 , pl. 10 , fig. 5 .

1971. Crassostrea gigas. Kuroda, Habe and Oyama, Sea Shells Sagami Bay, p. 596, pl. 86, fig. 1 .

1973. Ostrea (Crassostrea) gigas. NodA, Saito Ho-on Kai Mus., Res. Bull., no. 42 , pl. 5, fig. 6 .

1973. Crassostrea gigas. Oyama, Palaeont. Soc. Japan. Spec. Paper, no. 17, p. 89 , pl. 29 , figs. $1 \mathrm{a}-\mathrm{b}, 4 \mathrm{a}-\mathrm{b}$, pl. 30 , figs. $2 \mathrm{a}-\mathrm{b}$.

1977. Crassostrea gigas. HABE, Syst. Moll. Japan, p. 108, pl. 20, fig. 6 . 
Remarks:-Crassostrea gigas (THUNBERG) is a common species in Japanese waters, and it is generally characterized by its large-sized, elongated and fairly thick shell. The specimens in hand are quite identical to C. gigas.

Occurrence:-Loc. 1 (Common).

Geologic distribution:-Tokyo, Yurakucho and Tokumaru Formations, Tokyo Metropolis; Sakuragicho, Yokosuka, Koshiba and Kuratsubo Formations, Kanagawa Prefecture; Kotori Formation, Chiba Prefecture; Nanakita, Gobanshoyama, Tatsunokuchi and Kama Formations, Miyagi Prefecture; Ogawa and Shigarami Formations, Nagano Prefecture; Omma Formation, Ishikawa Prefecture; Konobe Formation, Mie Prefecture; Noheji Formation, Aomori Prefecture; Shishinai Formation, Hokkaido; Byoritsu and Shokkozan Formations, Taiwan.

Recent distribution:-Sakhalin, Hokkaido, Honshu, Shikoku, Kyushu, Korea, China and Southeast Asia.

Geologic range:-Miocene to Recent.

\author{
Family Chamidae \\ Genus Chama LiNNAEUS, 1758 \\ Chama japonica LAMARCK, 1819 \\ Pl. 4, Figs. 1a-b
}

1871. Chama ambigua Lischke. Japan Meer. Conch., vol. 2, p. 128, pl. 8, fig. 2. 1977. Chama japonica LAMARCK. HABE, Syst. Moll. Japan, p. 137.

Remarks:-Several rather well preserved specimens were examined. The present species is characterized by very thick, rather large, suborbicular shell with distinct, coarse lamellated concentric wavy ridge, gently curved, rather shallow furrow running from beak to anterior dorsal margin and smooth interior ventral margin.

The present fossil record may be the first from Japan.

Dimensions (in $\mathrm{mm}$ ) :-

$\begin{array}{llllll}\text { Height } & 60 & 38 & 34.5 & 30.5 & 31 \\ \text { Length } & 55 & 33.5 & 33.5 & 24 & 28.5 \\ \text { Depth } & 24 & 14 & 14 & 10 & 13\end{array}$

Occurrence:-Loc. 5 (Common).

Recent distribution:-Southern Japan.

Geologic range:-Pliocene to Recent.

Family Carditidae

Genus Cyclocardia CoNRAD, 1867

Cyclocardia sp. cf. C. ferruginea (CLESsin, 1888)

Pl. 4, Fig. 2

Compared with:

1888. Venericardia ferruginea Clessin. Syst. Conch. Cab., vol. 10, pt. 1, p. 17, pl. 6, fig. 11 . 
Remarks:-One small specimen was examined. The present one can be distinguished from Cyclocardia ferruginea, a Recent species of Japan, by its less orbicular shell about 21, granulated radial ribs which are nearly equal to or more or less narrower than their interspaces in breadth and not well impressed lunule. Therefore, it may represent a new form, but naming is withheld until more specimens are examind.

Dimensions (in $\mathrm{mm}$ ) :- Height 10.2, length 10.2, depth 3.8 .

Occurrence:-Loc. 3 (Rare).

Genus Megacardita SACC0, 1899

Megacardita granulicostata (NOMURA, 1933)

Pl. 4, Figs. 3, 4, 5, 6

1928b. Venericardia cipangoana YoKoyama. Imp. Geol. Surv. Japan, Rep., no. 101 , p. 86, pl. 9, figs. 3-5.

1933. Venericardia granulicostata Nomura. Sci. Rep., Tohoku Imp. Univ., 2nd Ser., vol. 16, no. 1, p. 70, pl. 2, figs. 7a-d.

1957b. Venericardia (Megacardita) granulicostata. Sнuто, Mem. Fac. Sci., Kyushu Univ., Ser. D, vol. 6, no. 2, p. 87, pl. 22, figs. 17, 18.

1980. Venericardia (Megacardita) granulicostata. NoDA, Sci. Rep. Inst. Geosci., Univ. Tsukuba, Ser. B, vol. 1, p. 86, pl. 3, figs. 10a-b.

Remarks:-The present species is characterized by its oblong-ovate, moderately inflated, rather thick shell and about 15, distinct, granulated radial ribs. Megacardita ferruginosa (ADAMS and REEVE), a Recent species of Japan, can be distinguished from the present species by its low radial ribs which are smooth on surface.

$\begin{array}{lccrrrr}\text { Dimensions (in mm) :- } & & & & \\ \text { Valve } & \text { Right } & \text { Right } & \text { Right } & \text { Left } & \text { Left } & \text { Left } \\ \text { Height } & 19.5 & 18.0 & 17.0 & 21.0 & 20.0 & 19.0 \\ \text { Length } & 24.5 & 23.0 & 21.0 & 26.2 & 24.5 & 23.6 \\ \text { Depth } & 7.5 & 6.0 & 6.2 & 7.0 & 7.8 & 6.4\end{array}$

Occurrence:-Loc. 2 (Few); Loc. 5 (Abundant).

Geologic distribution:-Byoritsu Formation, Taiwan; Takanabe Member of Koyu Formation, Miyazaki Prefecture; Shinzato Formation, Okinawa Prefecture.

Geologic range:-Pliocene.

\author{
Family Crassatellidae \\ Genus Bathytormus STEWART, 1930 \\ Bathytormus foveolatus (SOWERBY, 1870) \\ Pl. 4, Figs. 7a-b
}

1927. Crassatellites yagurai Makiyama. Mem. Coll. Sci., Kyoto Imp. Univ., Ser. $B$, vol. 3 , no. 1 , p. 38 , pl. 2 , figs. $1-4$.

1928b. Cryptogramma kaneharai YoкоYAмA. Imp. Geol. Surv. Japan, Rep. no. 101, p. 80 , pl. 8 , fig. 5 . 
1928. Crassatellites oinouyei YокоҮама. Ibid., no. 101, p. 89, pl. 13, figs. 1-3.

1929. Crassatellites yagurai. YoкоYамA, Ibid., no. 104, p. 15, pl. 8, fig. 3.

1960. Crassatellites foveolatus (Sowerby). MakiYama, Palaeont. Soc. Japan, Spec. Paper, no. 6, pl. 99, figs. 1-3, pl. 115, fig. 3.

1977. Bathytormus foveolatus. HABE, Syst. Moll. Japan, p. 163, pl. 30, figs. $12,13$.

Remarks:-One well preserved specimen was examined. This is characterized by its small, rather thick shell with coarse concentric cords, a ridge running from beak to posterior corner and fine marginal serration in interior surface.

Dimensions (in $\mathrm{mm}$ ) :-Right valve, height 6.0, length 8.0, depth 2.0 .

Occurrence:-Loc. 5 (Rare).

Geologic distribution:-Tenno Formation, Shizuoka Prefecture; Ananai Formation, Kochi Prefecture; Byoritsu Formation, Taiwan.

Recent distribution:-Taiwan and Southwest Pacific.

Geologic range:--Pliocene to Recent.

\author{
Family Cardiidae \\ Genus Vepricardium IREDALE, 1929 \\ Vepricardium sp.
}

Remarks:-One imperfect specimen was examined. The present specimen is small and its external surface is sculptured with about 30, distinct, squarish, spinose radial ribs which somewhat broader than their interspaces with more or less lamellated fine concentric lines. Although the hinge teeth are not observed, it is reasonably referred to Vepricardium IREDALE, 1929 on the basis of the above noted features.

Dimensions (in $\mathrm{mm}$ ):- Height 8.5, length 10.0, depth 4.2 .

Occurrence:-Loc. 5 (Rare).

Genus Vasticardium IREDALE, 1927

Vasticardium sp.

Remarks:-One imperfect specimen was examined. The shell is small, thin, longer than high, moderately inflated, and its external surface is sculptured with numerous smooth radial ribs which are nearly equal to their interspaces in breadth. Although the hinge teeth are not observed, it is reasonably referred to Vasticardium IREDALE, 1927 on the basis of the above noted features.

Dimensions (in mm) :-Height 10.0, length 11.0, depth ca. 4.0.

Occurrence:-Los. 4 (Rare).

\title{
Genus Fulvia GraY, 1853 \\ Fulvia sp.
}

Remarks:-One imperfect specimen. The present specimen is characterized by its thin, inflated shell. Radial shallow furrows on the shell are distinct.

Occurrence:-Loc. 5 (Rare). 


\author{
Family Tellinidae \\ Genus Macoma LEACH, 1819 \\ Macoma sp.
}

Remarks:-A few imperfect specimens were examined. Shell small, thin, slightly inflated; posterior side broadly rounded; sculpture of fine concentric growth lines and blunt ridge running from beak to posterior corner.

Occurrence:-Loc. 2 (Few).

Genus Psammotreta DALL, 1900

Subgenus Pseudometis LAMY, 1918

Psammotreta (Pseudometis) praerupta (SALISBURY, 1934)

P1. 4, Fig. 8

1952. Pseudometis praerupta (SALISBURY). HABE, Gen. Japan. Shells, p. 221, figs. 558, 559.

1961. Pseudometis praerupta. HAyASaKa, Sci. Rep., Tohoku Univ. 2nd Ser., vol. 33 , no. 1 , p. 59 , pl. 7 , figs. $6 a-b$.

1971. Macoma (Pseudometis) praerupta. HAYASAKA and OKI, Rep. Fac. Sci., Kagoshima Univ., no. 4, p. 7, pl. 1, fig. 6.

1977. Psammotreta (Pseudometis) praerupta. HABE, Syst. Moll. Japan, p. 212, pl. 43 , figs. 8,9 .

Remarks:-Several rather ill preserved specimens were examined. The present specimens are characterized by its medium-sized, ovate, slightly inflated, thin shell with fine concentric growth lines, posteriorly situated beak, rounded anterior side, more or less truncated posterior side, rather distinct ridge running from beak to posterior corner and deep pallial sinus.

Dimensions (in $\mathrm{mm}$ ) :-Left valve: height 40.0, length 52.5 ; right valve: height ca. 30.0 , length 40.5 .

Occurrence:-Loc. 2 (Common).

Geologic distribution:-Kogashira Formation, Kagoshima Prefecture; Toshima Formation, Aichi Prefecture.

Recent distribution:-From Kii Peninsula to the Philippines.

Geologic range:-Pliocene to Recent.

Family Solecurtidae

Genus Solecurtus BlainviLle, 1824

Solecurtus miyakojimaensis MASUdA, SATo and ShUto, n. sp.

Pl. 4, Figs. 9a-b

Holotype:-GK-L 11828.

Type locality:-Loc. 2. ôra Formation.

Diagnosis:-Shell generally large, broadly rounded at anterior side and obliquely truncated at posterior side. Beak situated anteriorly at about onethird of shell length.

Description:- Shell rather large, rather thin, slightly inflated, subrectangle 
in outline, longer than high, broadly rounded at anterior side and obliquely truncated at posterior side; open at both ends; beak situated at about anterior one-third of shell length; ventral and dorsal margins straight, nearly parallel with each other. Surface with fine concentric growth lines and diverging numerous, somewhat lamellate, low but distinct sulci which are nearly vertical with ventral margin in middle to posterior area but $\mathrm{V}$ shape at anterior area. Interior characters remain unknown.

Comparison:-This species can be distinguished from Solecurtus divaricatus (LISCHKE, 1869), a Recent species of Japan and Western Pacific, by its anteriorly situated beak and characteristic surface sculpture. The present species is closely similar to S. rhombus (SPENGLER, 1802) living in the southern Japanese waters to Southeast Asian waters in the general feature, but it is distinguished from the latter in having somewhat less rectangular outline and coarser diverging sulci.

Dimensions (in $\mathrm{mm}$ ) :-Height 31.0, length 69.0, depth 21.0 (Holotype, intact valve); height 16.0, length 39.0 .

Occurrence:-Few.

\author{
Genus Azorinus RECLUZ, 1869 \\ Azorinus abbreviatus (GouLd, 1861) \\ Pl. 4, Figs. 10, 11, 12
}

1861. Solecurtus abbreviatus Gound. Proc. Boston Soc. Nat. Hist., vol. 8, p. 26.

1874. Solecurtus abbreviatus. REEve, Conch. Icon., vol. 19, Solecurtus, sp. 6, pl. 2 , figs. $6 \mathrm{a}-\mathrm{b}$.

1888. Solecurtus abbreviatus. Clessin in Martini und Chemnitz, Syst. Conch. Cab., vol. 11, pt. 3, p. 93, pl. 22, fig. 3 .

1920. Solecurtus abbreviatus. Yokoyama, Jour. Coll. Sci., Imp. Univ. Tokyo, vol. 39 , art. 6 , p. 111 , pl. 7, figs. $12,13$.

1954. Azorinus abbreviatus. TAKI and Oyama, Palaeont. Soc. Japan, Spec. Paper, no. 2, pl. 8, figs. 12, 13.

1970. Azorinus abbreviatus. HAYASAKA and HaShimoto, Rep. Fac. Sci., Kagoshima Univ., no. 3, p. 20, pl. 1, figs. 10, 11.

1971. Azorinus (Azorinus) abbreviatus. ShUто, Mem. Fac. Sci. Kyushu Univ., Ser. $D$, vol. 21 , no. 1 , p. 51-52, pl. 6, figs. 17,18 and text-fig. 11.

1973. Azorinus abbreviatus. Oyama, Palaeont. Soc. Japan, Spec. Paper, no. 17, p. 111, pl. 54 , figs. 4,5 .

1977. Azorinus abbreviatus. HABE, Syst. Moll. Japan, p. 225, pl. 47, figs. 9, 10.

Remarks:-The present specimens are characterized by their rather small, thin, compressed, transversely oblong shell with coarse concentric cords and fine concentric growth lines, and a shallow and rather wide radial groove running from beak to middle part of ventral margin. They quite conform with Azorinus abbreviatus (GouLD) living in the southern Japanese waters to the equatorial seas.

Dimensions (in $\mathrm{mm}$ ) :-Right valve: height 15.0 , length 31.5 ; right valve: height 12.5, length 24.0; left valve: height 14.5, length 31.5 .

Occurrence:-Loc. 2 (Few). 
Geologic distribution:-Naganuma Formation, Kanagawa Prefecture; Onejime Formation, Kagoshima Prefecture.

Recent distribution:-Southern Japan and Southwest Pacific.

Geologic range:-Pliocene to Recent.

Family Glossidae

Genus Meiocardia H. and A. ADAMs, 1857

Meiocardia vulgaris (REEVE)

Pl. 4, Figs. 13a-b, 14a-b, 15a-b

1927. Meiocardia vulgaris ReEve. Fischer. Palaeont. v. Timor, Lief. 15, p. 121.

Remarks:-The present species is characteriezd by the following features:Shell medium in size, rather thick, oblong ovate in outline, longer than high, moderately inflated; anterior side narrowly rounded; posterior side truncated behind; ventral margin broadly rounded; beak pointed, strongly incurved anteriorly. Valve with distinct, blunt keel running from beak to somewhat protruded posterior ventral corner, making elongated, somewhat concaved postero-dorsal area. Surface with more or less regularly spaced, low, flat-topped, coarse concentric cords which are much broader than their interspaces and terminate upon keel, but with only fine concentric lines at posterodorsal area. Ligament broadly arched; anterior cardinals short; posterior one long, gently curved. Interior ventral margin smooth.

The present specimens are quite identical to Meiocardia vulgaris (REEVE, 1845) living in Okinawa and southern waters. It is readily distinguished from Meiocardia tetragona (ADAMS and REEVE), a Recent species of Southern Japan, by its moderately inflated high shell, strongly incurved beak and low, flat coarse concentric cords. Also Meiocardia moltkiana SPENGLER, a Recent species of Southern Japan, is distinguishable from the present one by its greatly inflated shell, distinct, coarse concentric cords, much prominent keel and very protruded posterior ventral corner.

Dimensions (in $\mathrm{mm}$ ) :-

$\begin{array}{lcccc}\text { Valve } & \text { Right } & \text { Right } & \text { Right } & \text { Left } \\ \text { Height } & 19.2 & \text { ca. } 19.0 & 16.0 & 8.0 \\ \text { Length } & 23.2 & 24.5 & 19.0 & 9.5 \\ \text { Depth } & 9.0 & - & 8.0 & 4.5\end{array}$

Occurrence:-Loc. 2 (Rare); Loc. 3 (Common).

Recent distribution:-South of Philippines.

Geologic range:-Pliocene to Recent.

Family Veneridae

Genus Venus Linnaeus, 1758

Subgenus Ventricoloidea SACCo, 1900

Venus (Ventricoloidea) foveolata SOWERBY, 1853

Pl. 5, Figs. 2, 3, 4 
1853. Venus foveolata Sowerby. Thes. Conch., vol. 2, p. 730, pl. 154, fig. 46.

1863. Venus foveolata. ReEve, Conch. Icon., vol. 14, Venus, sp. 11, pl. 4, fig. 11.

1926. Chione casinaeformis Yokочама. Jour. Fac. Sci., Imp. Univ., Tokyo, Sec. C, vol. 1, pt. 9, p. 352, pl. 39, figs. 7-9.

1926. Chione casinaeformis. YokoYama, Ibid., vol. 1, pt. 9, p. 368, pl. 42, figs. 3 , 3 a.

1928b. Chione casinaeformis. YokоYама, Imp. Geol. Surv. Japan, Rep., no. 101, p. 79 , pl. 8, fig. 4 .

1951. Venus (Ventricola) foveolata. HABE, Gen. Japan. Shells, no. 2, p. 171, fig. 382 .

? 1957. Ventricola foveolata miyazakiensis Shuto, Jour. Geol. Soc. Japan, vol. 63, no. 745 , p. 569 , text-fig. 4 .

1958. Ventricoloidea foveolata. Makiyama, Palaeont. Soc. Japan, Spec. Paper, no. 4, pl. 51, figs. 7-9, pl. 54, figs. 3, 3a.

1958. Venus (Ventricoloidea) foveolata. HABE, Publ. Seto Mar. Biol. Lab., vol. 7, no. 1, p. 37, pl. 2, fig. 20.

? 1960. Venus (Ventricoloidea) foveolata miyazakiensis. Shuto, Mem. Fac. Sci., Kyushu Univ., Ser. D, vol. 9, no. 3, p. 129, pl. 14, figs. 13, 16, text-figs. 8, 11, 13.

1960. Ventricoloidea foveolata. Makiyama, Palaeont. Soc. Japan, Spec. Paper, no. 6 , pl. 94 , fig. 4 .

1966. Ventricoloidea foveolata. Aoki, Trans. Proc. Palaeont. Soc. Japan, N. S., no. 62 , p. 255 , pl. 31 , fig. 8 .

1970. Venus (Ventricola) foveolata. Hayasaka and Hashimoto, Rep. Fac. Sci., Kagoshima Univ., no. 3, p. 20, pl. 31, fig. 8.

1971. Venus (Ventricoloidea) foveolata. Kuroda, HABE and Oyama, Sea Shells Sagami Bay, p. 659, pl. 93, fig. 2.

1977. Ventricolaria foveolata. HABE, Syst. Moll. Japan, p. 246, pl. 51, fig. 1.

1980. Venus (Ventricoloidea) foveolata. NodA, Sci. Rep. Inst. Geosci., Univ. Tsukuba, Sec. B, vol. 1, p. 90, pl. 3, figs. $12 \mathrm{a}-\mathrm{b}, 19$.

Remarks:-The present specimens are characterized by their medium sized, rather thick, suborbicular shell which is longer than high, broadly curved, long postero-dorsal margin, pointed beak, regularly and distantly spaced rather prominent concentric lamellae, two or more, fine, more or less lamellated con-

\section{Explanation of Plate 5}

(All figures in natural size unless otherwise stated)

Fig. 1. Clementia papyracea (GRAY). Loc. 2. ..................42

Figs. 2, 3, 4. Venus (Ventricoloidea) foveolata SowerBy. 4, ×1.5. Loc. 5 . . 35

Figs. 5, 6, 7, 8. Ventricolaria toreuma (GoULD). $\times 1.5$. Loc. 5. . . . . ...37

Figs. 9, 10. Dosinia (Paradosinia) amphidesmoides (REEVE). Loc. 2. .....39

Fig. 11. Meretrix sp. cf. M. lusoria (RönING). Loc. 1. . . . . . . . . . . .41

Figs. 12, 13. Cyclina sp. cf. C. sinensis (GMELIN). Loc. 1. ..........41

Figs. 14, 15, 16 . Paphia exilis Shuto, Loc. 2. .................40

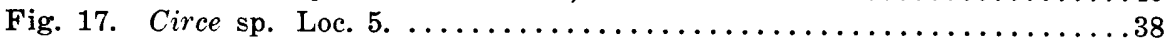

Figs. 18a-b, 19. Lioconcha (Sulcilioconcha) philippinarum (HANLEY).

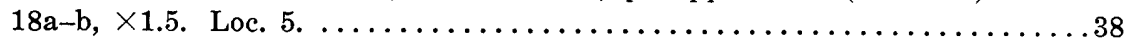

Figs. 20, 21, 22. Anisocorbula tosana (Yокочама). $\times 1.5$. Loc. 2 . . . ...43

Figs. 23a-b. Anisocorbula socialis (MARTIN). $\times 1.5$. Loc. 5. ..........43

Fig. 24. Myadora ikebei HABE. $\times 3$. Loc. 5 . .................. 44 


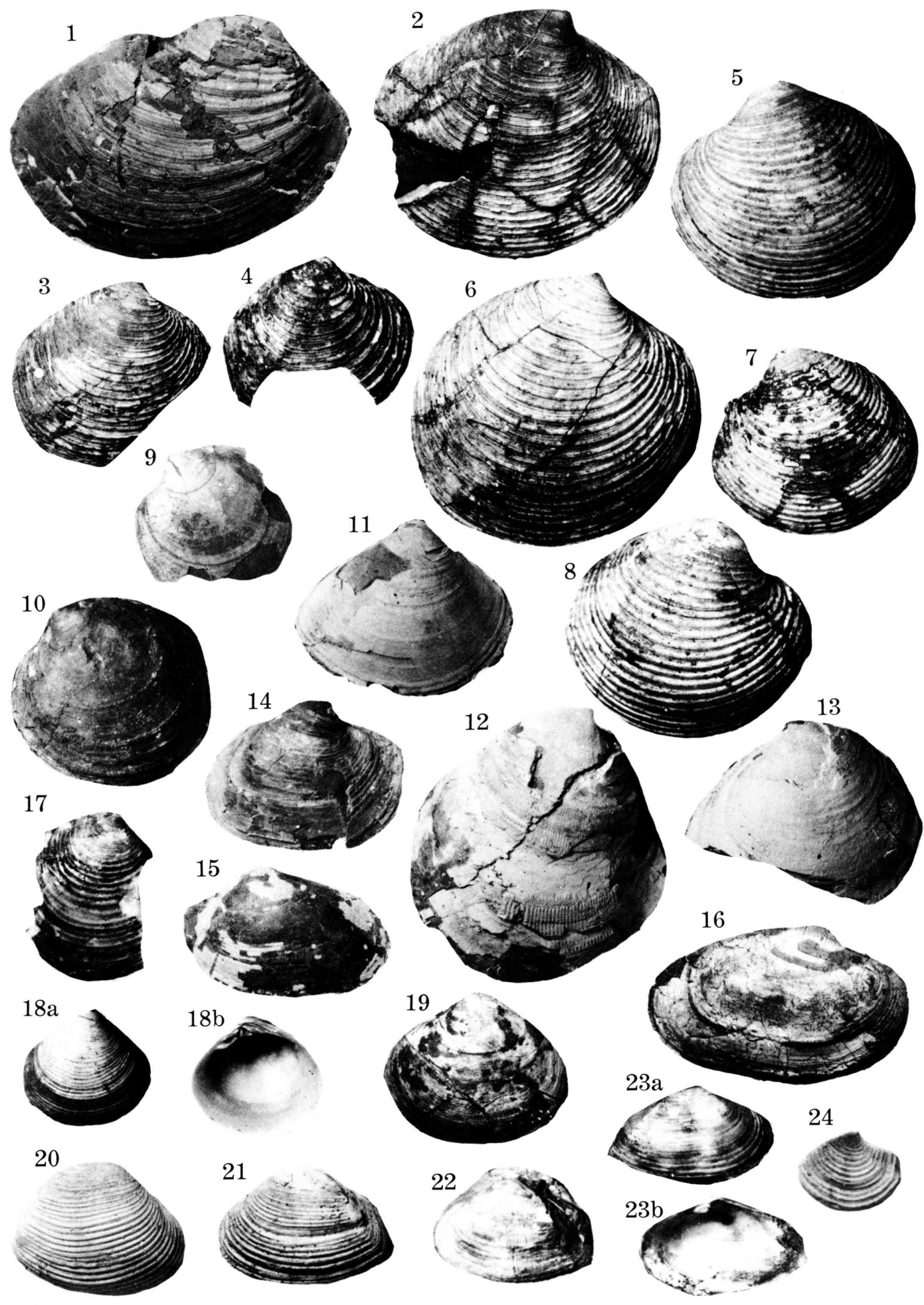

Y. SATo et al.: Pelecypod Fauna of the Shimajiri Group in Miyako-jima 
centric lines in interspaces between main concentric lines, narrowly incised escutcheon, rather narrow and long, not well defined shallow lunule and finely crenulated internal ventral margin.

NoDA (1980) included Venus toreuma illustrated by Nomura and ONISI (1940) from the Miocene Murata Formation in Miyagi Prefecture, into the present species, but NoMURA and ONIsI's specimen can be distinguished from the present one by its rather short postero-dorsal margin and surface sculpture. Venus (Ventricoloidea) foveolata miyazakiensis SHUTO (1957) from the Lower part of Miyazaki Group is smaller and less orbicular than the normal form, but the difference is so slight that it is questionable whether that taxon is valid or not. Further study is necessary to settle the problem.

Dimensions (in $\mathrm{mm}$ ) :-

$\begin{array}{lccccr}\text { Valve } & \text { Right } & \text { Right } & \text { Right } & \text { Right } & \text { Left } \\ \text { Height } & 39.0 & 29.0 & 27.0 & 17.0 & 11.5 \\ \text { Length } & 44.0 & 31.0 & 31.0 & 20.0 & 13.0 \\ \text { Depth } & 10.0 & 9.0 & 8.0 & 5.0 & 4.0\end{array}$

Occurrence:-Loc. 3 (Rare); Loc. 5 (Common).

Geologic distribution:-Shinzato Formation, Okinawa Prefecture; Miyazaki Group, Miyazaki Prefecture; Ananai Formation, Kochi Prefecture; Sagara Formation, Shizuoka Prefecture; Onejime Formation, Kagoshima Prefecture; Byoritsu Formation, Taiwan.

Recent distribution:-Honshu (Boso Peninsula as north limit), Shikoku, Kyushu and East China Sea.

Geologic range:-Late Miocene to Recent.

Subgenus Ventricolaria KEEN, 1954

Venus (Ventricolaria) toreuma (GoULd, 1850)

Pl. 5, Figs. 5, 6, 7, 8

1863. Venus toreuma Gould. REEve, Conch. Icon., vol. 14, Venus, sp. 64, pl. 16, figs. 64a-b.

1924. Venus toreuma. Yokoyama. Jour. Coll. Sci., Imp. Univ. Tokyo, vol. 45, art. 1, p. 44, pI. 2, fig. 22.

1954. Venus (s.s.) toreuma. TAKI and Oyama, Palaeont. Soc. Japan, Spec. Paper, no. 2, pl. 39, fig. 22.

1958b. Venus (Ventricolaria) toreuma. HABE, Publ. Seto Mar. Biol. Lab., vol. 7, no. 1 , p. 36 , pl. 2 , fig. 8.

1971. Venus (Ventricolaria) toreuma. KUROdA, HABE and OyAma, Sea Shells Sagami Bay, p. 658, pl. 93, fig. 11.

1973. Ventricolaria toreuma. Oyama, Palaeont. Soc. Japan, Spec. Paper, no. 17, p. 103 , pl. 46 , fig. 3 .

1977. Venus (Ventricoloidea) toreuma. HABE, Syst. Moll. Japan, p. 246, pl. 51, figs. $2,3$.

Remarks:-The present species is characterized by its rather small, orbicular, thick, inflated shell with regularly spaced broad, low, rounded concentric lines and regularly spaced fine concentric lines in interspaces between main con- 
centric lines, somewhat granulated concentric lines near anterior and posterodosal margins by radial threads, ditinctly impressed small lunule, shallow, triangular pallial sinus and faint, fine serration at internal ventral margin.

NoMURA and ONISI (1940) illustrated the present species from the Miocene Murata Formation, Miyagi Prefecture, but the examination of the present writers suggests that the Murata specimen (Saio Ho-on Kai Mus., Nat. Hist., Reg. no. 21676) may represent a new form, because it is longer than high and less inflated than that of the present species.

Dimensions (in $\mathrm{mm}$ ):-

$\begin{array}{lcccccr}\text { Valve } & \text { Right } & \text { Right } & \text { Right } & \text { Left } & \text { Left } & \text { Left } \\ \text { Height } & 27.4 & 23.3 & 23.0 & 30.5 & 28.0 & 23.0 \\ \text { Lengtn } & 31.0 & 26.0 & 26.0 & 33.0 & 30.0 & 25.5 \\ \text { Depth } & 10.2 & 8.5 & 9.0 & 11.8 & 10.6 & 8.5\end{array}$

Occurrence:-Loc. 5 (Abundant).

Geologic distribution:-Numa Coral Bed, Chiba Prefecture.

Recent distribution:-Honshu (Boso Peninsula as north limit), Shikoku, Kyushu and Tropical Pacific Region.

Geologic range:-Pliocene to Recent.

\section{Genus Glycydonta CotToN 1936 \\ Glycydonta ? sp.}

Remarks:-One small fragmental specimen having distinct radial threads and concentric growth lines on surface was examined.

Occurrence:-Loc. 5 (Rare).

Genus Circe SchumaCher, 1817

Circe sp.

Pl. 5, Fig. 17

Remarks:-One fragmental specimen was examined. Shell is mediumsized, thick, hardly inflated umbonal region with rather pointed small beak. Heterodont cardinal teeth devoid of posterior lateral tooth. It is similar to Circe scripta (LINNAEUS), but the concrete identification is withheld.

Occurrence:-Loc. 5 (Rare).

Genus Lioconcha Mörch, 1853

Subgenus Sulcilioconcha HABE, 1951

Lioconcha (Sulcilioconcha) philippinarum (HANLEY, 1844)

P1. 5, Figs. 18a-b, 19

1844. Cytherea philippinarum Hanley. Proc. Zool. Soc. London, for 1844. p. 110.

1851. Cytherea philippinarum. SowERBY, Thes. Conch., vol. 2, p. 627, pl. 136, fig. 176.

1864. Cytherea philippinarum. ReEve. Conch. Icon. vol. 14, Cytherea, pl. 10, figs. $47 \mathrm{a}, \mathrm{b}$. 
1869. Venus (Cytherea-Cione) philippinarum. RöMER. Monogr. Venus, Bd. 1, p. 139 , pl. 37 , figs. 2a-e.

1920. Cytherea (Lioconcha) molengraaffi. TISch, Palaeont. von Timor, Lief. 8, p. 108, pl. 139, figs. 273 and 274.

1932. Hysteroconcha (? Lamelliconcha) philippinarum. PRASHAD, Siboga-Expeditie. Monogr. 53c, p. 217, pl. 6, figs. 7-10.

1951. Lioconcha (Sulcilioconcha) philippinarum. HABE, Gen. Japan. Shells, no. 2 , p. 158 , figs. $364-365$.

1971. Lioconcha (Sulcilioconcha) philippinarum. Shuto, Mem. Fac. Sci., Kyushu Univ., Ser. D, Geol., vol. 21, no. 1, p. 54, pl. 9, figs. 7, 9, 11, 13 and 14.

1977. Lioconcha (Sulcilioconcha) philippinarum. HABE, Syst. Moll. Japan, p. 259 , pl. 52, figs. 12,13 .

1982. Lioconcha (Sulcilioconcha) philippinarum. KANNo, OHARA and CAAGUSAN. Cont. Geol. and Paleont. Southeast Asia CCXXII, vol. 24, p. 84, pl. 15, figs. 14a-b.

Remarks:-Two well preserved specimens were examined. The present species is distinguished from the other species by its rather small, thick, inflated shell with hardly curved dorsal margins, sculpture of distinct and regular concentric lines, indistinct lunule, very shallow pallial sinus and strong anterior lateral tooth. They are quite identical with Lioconcha (Sulcilioconcha) philippinarum.

The present record may be the first from the Pliocene formation in Japan.

Dimensions (in mm) :-Left valve, height 24.5, length 28.0, depth 9.0; right valve, height 12.0 , length 13.5 , depth 4.2 .

Occurrence:-Loc. 5 (Few).

Recent distribution:-Southern Japan (Amami Islands as north limit), Taiwan and the Philippines.

Geologic range:-Pliocene to Recent.

Genus Dosinia Scopol, 1777

Subgenus Pardosinia IREDALE, 1929

Dosinia (Pardosinia) amphidesmoides (REEVE, 1850)

Pl. 5, Figs. 9, 10

1850. Artemis amphidesmoides REEve. Conch. Ichon., vol. 6, Artemis, sp. 48, pl. 8 , fig. 48.

1855. Artemis amphidesmoides. Sowerby, Thes. Conch., vol. 2, p. 659, pl. 141, fig. 19.

? 1928. Dosinia angulosa (PhiliPri). Yokoyama, Imp. Geol. Surv. Japan, Rep., no. 101, p. 75 , pl. 7 , fig. 4 .

1933. Dosinia amphidesmoides. NomUra, Sci. Rep., Tohoku Imp. Univ., 2nd Ser., vol. 16 , no. 1 , p. 96 , pl. 4 , fig. 18.

?1960. Dosinia angulosa. MakiYama, Palaeont. Soc. Japan, Spec. Paper, no. 6, pl. 93, fig. 4.

1961. Dosinia (Pardosinia) amphidesmoides. HABE, Colored Illust. Shells Japan, p. 131 , pl. 59 , fig. 9 .

Remarks:-Rather well preserved two intact and two isolated shells were examined. The present specimens are characterized by the following features. 
Shell small, thin, moderately inflated, orbicular but with blunt angulation at about middle of postero-dorsal margin. Escutchen rather distinct, lunule small. It is reasonably identified with Dosinia (Pardosinia) amphidesmoides (REEVE).

The present species resembles Dosinia (Dosinella) angulosa (PHILIPPI), a Recent species of Southern Japan, but it can be distinguished from angulosa by its small, orbicular, thin shell, small lunule and indistinct escutcheon.

Although Yokoyama (1928) described Dosinia angulosa from the Byoritsu Formation, Taiwan, judging from figure YokoYAMA's angulosa may be referred to the present species. But further study is necessary to settle this problem.

Dimensions (in $\mathrm{mm}$ ) :-Intact valve: height 31.3 , length 31.5 , depth 13.5; intact valve: height 26.0, length 26.5, depth 11.5.

Occurrence:-Loc. 2 (Few).

Geologic distribution:-Byoritsu Formation, Taiwan.

Recent distribution:-Southern Japan (Kii Peninsula as north limit), Taiwan and Philippines.

Geologic range:-Pliocene to Recent.

\section{Dosinia sp.}

Remarks:-One unfavorably preserved left valve was examined. It is large, rather thick shell.

Dimensions (in $\mathrm{mm}$ ) :-Left valve, height 60.6, length 63.5.

Occurrence:-Loc. 1 (Rare).

Genus Paphia RöDING, 1798

Paphia (Paphia) exilis Shuto, 1957

Pl. 5, Figs. 14, 15, 16

1957. Paphia (Paphia) exilis exilis Shuto. Japan. Jour. Geol. Geogr., vol. 28, nos. 1-3, p. 142, pl. 12, figs. 3 , 4.

1961. Paphia (Paphia) exilis exilis. ShUto. Mem. Fac. Sci., Kyushu Univ., Ser. $D$, vol. 10, no. 2, text-fig. 19-1.

1969. Paphia (Paphia) exilis exilis. HAYASAKa, Rep. Fac. Sci., Kagoshima Univ., no. 2, p. 46 , pl. 3, fig. 1 .

Remarks:-Several unfavorably preserved specimens were examined. Shell rather small, oblong, longer than high, moderately inflated; beak pointed, situated at about one third anteriorly. Surface with regularly spaced, numerous, distinct, flatly round-topped concentric cords which are somewhat broader than their shallow interspaces. The specimens show shorter and larger forms. They are respectively identical with Paphia exilis abbreviata SHuto and $P$. (P.) exilis exilis SHUTo.

Paphia euglypta (PhILIPPI), a common Recent species of Japan, can be distinguished from the present one by its coarser, more prominent, very wide concentric cords and deep and wide interspaces between concentric cords.

Dimensions (in $\mathrm{mm}$ ) :-Right valve: height 22.6 , length 37.5 , depth ca. 8.5 ; right valve: height 19.0 , length 30.2 , depth ca. 
Occurrence:-Loc. 2 (Common); Loc. 3 (Few).

Geologic distribution:-Miyazaki Group, Miyazaki Prefecture.

Geologic range:-Pliocene.

Genus Meretrix LAMARCK, 1799

Meretrix sp. cf. M. lusoria (RöDING, 1798)

Pl. 5, Fig. 11

Compared with:-

1971. Meretrix lusoria (Röding). Kunoda, Habe and Oyama, Sea Shells Sagami Bay, p. 647, pl. 94, figs. 3-6.

Remarks:-Two imperfect specimens were examined. Shell medium in size, subtriangular, moderately inflated; beak pointed, situated somewhat anteriorly; surface rather smooth but with fine concentric growth lines. Meretrix meretrix (Linnaeus, 1758), a Recent species of Western Pacific (Okinawa as north limit) resembles the present species but it can be distinguished from the latter by its rounded and inflated shell.

Dimensions (in $\mathrm{mm}$ ) :-Right valve: height 27.0 , length 32.5 , depth ca. 7.5 ; left valve: height 28.0, depth ca. 8.0.

Occurrence:-Loc. 1 (Rare).

Genus Cyclina Deshayes, 1850

Cyclina sp. cf. C. sinensis (GMELIN, 1791)

Pl. 5, Figs. 12, 13

Compared with:-

1971. Cyclina sinensis (Gmelin). Kuroda, Habe and Oyama, Sea Shells Sagami Bay, p. 652, pl. 91, fig. 6 .

Remarks:-Two rather ill preserved specimens were examined. Shell large, suborbicular, inflated; surface with fine network by numerous fine radial threads and fine concentric growth lines.

OYAMA $(1950,1973)$ stated that Cyclina orientalis SowERBY, a Recent species of Japan, is to be separated from Cyclina sinensis (GMELIN) which ranges from Taiwan to Okinawa Islands. Also, KAMADA (1952), HAYASAKA (1961) and HAYASAKA and OKI (1971) pointed out that Cyclina orientalis corresponds to a part of the so-called sinensis and differs therefrom in its rounder outline and more solid shell. However, KURODA, HABE and OYAMA (1971) and HABE (1977) stated that Cyclina orientalis should be included into a synonym of Cyclina sinensis.

Dimensions (in $\mathrm{mm}$ ) :-Right valve: height 44.0, length 39.0, depth 16.0; left valve: height ca. 42 , length 38.0 , depth ca. 14.0 .

Occurrence:-Loc. 1 (Few). 
Genus Clementia GraY, 1842

Clementia papyracea (GRAY, 1825)

Pl. 5, Fig. 1

1855. Clementia paypracea (GRAY). Sowerby, Thes. Conch., vol. 2, p. 700, pl. 151, fig. 155.

1913. Clementia papyracea. Sмiтh, PhILIP. Jour. Sci., vol. 8, sec. A, no. 4, p. 272, pl. 7, fig. 5 .

1923. Clementia speciosa Yokoyama. Jour. Coll. Sci., Imp. Univ. Tokyo, vol. 45 , art. 2, p. 15, pl. 2 , figs. 14,15 .

1942. Clementia (Clementia) vatheleti MABILle. YABE and HataI, Japan. Jour. Geol. Geogr., vol. 18, nos. 1-2, p. 74, pl. 7, fig. 4.

1944. Clementia papyracea. OYAMA and SAKA, Bull. Shigen-kagaku Kenkyusho, vol. 1 , no. 2 , p. 141 , pl. 15 , figs. $15 a-b, 16$.

?1955. Clementia japonica MAsuda. Trans. Proc. Palaeont. Soc. Japan, N. S., no. 20 , p. 121 , pl. 19 , fig. 8.

1957. Clementia vatheleti. Makryama, Palaeont. Soc. Japan, Spec. Paper, no. 3, pl. 10, figs. 14, 15 .

1957. Clementia papyracea. Shuro, Jour. Geol. Soc. Japan, vol. 63, no. 745, text-fig. 2.

1960. Clementia papyracea. SнUто, Mem. Fac. Sci., Kyushu Univ., Ser. D, vol. 9 , no. 3 , p. 140 , pl. 13 , figs. 8,11 , text-figs. $5,6,9$.

1965. Clementia vatheleti. Kaseno and MatsuUra, Sci. Rep., Kanazawa Univ., vol. 10 , no. 1, pl. 16 , figs. 5,6 .

1970. Clementia papyracea. IWASAKI, Trans. Proc. Palaeont. Soc. Japan, N. S., no. 77 , p. 213, pl. 23 , fig. 5 .

1971. Clementia (Clementia) vatheleti. NodA, Ibid., no. 81, p. 42, pl. 7, fig. 13.

1971. Clementia (Clementia) papyracea. Shuro, Mem. Fac. Sci., Kyushu Univ., Ser. D, Geol., vol. 21, no. 1, p. 57, pl. 10, figs. 16, 18.

1974. Clementia papyracea. Itoigawa in Itoigawa, Shibata and Nishimoto, Bull. Mizunami Foss. Mus., no. 1, p. 93, pl. 27, figs. 6a-b.

1981. Clementia papyracea. Itoigawa, Shibata, Nishimoto and OKumura, Monogr., Mizunami Foss. Mus., no. 3-A, pl. 17, figs. 3a-b.

1982. Clementia papyracea. Kanno, Ohara and CaAgusan. Cont. Geol. and Palaeont. Southeast Asia, CCXXII, vol. 24, p. 89, pl. 15, fig. 15.

Remarks:-Three somewhat deformed specimens were examined. The present specimens are characterizd by its ovate, thin, inflated shell with coarse, irregularly spaced concentric cords and fine concentric growth lines in interspaces between coarse concentric cords and are identical with Clementia papyracea (GRAY).

As the present species usually tends to become deformed easily, it has hitherto been frequently confused with the other species. Therefore, it is considered that further detailed study on fossil and Recent specimens is necessary.

Dimensions (in $\mathrm{mm}$ ):-Right valve: height 35.5, length 49.0, depth 11.0; left valve: height ca. 41.0, length 51.0, depth ca. 12.0.

Occurrence:-Loc. 1 (Rare); Loc. 2 (Rare); Loc. 4 (Rare).

Geologic distribution:-Miocene Formations in Java and Philippines; Dainichi Formation, Shizuoka Prefecture; Nakoshi Formation, Okinawa Prefecture; Tsukiyoshi Formation, Gifu Prefecture; Miyazaki Group, Miyazaki Prefecture; Higashi-Innai Formation, Ishikawa Prefecture; Nakayama Formation, 
Fukushima Prefecture.

Recent distribution:-East China Sea and South China Sea.

Geologic range:-Miocene to Recent.

\author{
Family Corbulidae \\ Genus Anisocorbula IREDALE, 1930 \\ Anisocorbula tosana (Yокочама, 1929) \\ Pl. 5, Figs. 20, 21, 22
}

1929. Corbula tosana Yoкочама. Imp. Geol. Surv. Japan, Rep., no. 104, p. 15, pl. 8, fig. 1.

1949. Anisocorbula tosana. HABE, Illust. Catalogue Japan Shells, Erodonidae, p. 3, pl. 1, figs. 10, 11 .

1960. Aloides tosana. MakiYama, Palaeont. Soc. Japan, Spec. Paper, no. 6, pl. 115, fig. 1.

1980. Corbula (Caryocorbula) cf. tosana. NoDA, Sci. Rep. Inst. Geosci., Univ. Tsukuba, Ser. $B$, vol. 1, p. 93, pl. 3, figs. 18a-b.

Remarks:-The present specimens are characterized by their ovate, very thick, inflated, stumpy shell with coarse concentric cords and distinct ridge running from beak to posterior ventral corner.

HABE (1977) pointed out that the present species is a synonym of Anisocorbula scaphoides (HINDS), a Recent species of Southeast Asia, but it seems that Anisocorbula scaphoides can be distinguished from the present species by its thin, longer and less inflated shell and protruded posterior corner.

Dimensions (in $\mathrm{mm}$ ) :-

$\begin{array}{lcrrrrc}\text { Valve } & \text { Right } & \text { Right } & \text { Right } & \text { Left } & \text { Left } & \text { Intact v. } \\ \text { Height } & 14.0 & 14.0 & 13.0 & 13.0 & 13.0 & 11.5 \\ \text { Length } & 19.0 & 17.0 & 18.0 & 17.0 & 17.0 & 15.0 \\ \text { Depth } & 8.0 & 7.5 & 11.0 & 7.0 & 5.0 & 10.0\end{array}$

Occurrence:-Loc. 2 (Abundant); Loc. 3 (Few); Loc. 5 (Common).

Geologic distribution:-Ananai Formation, Kochi Prefecture; Shinzato Formation, Okinawa Prefecture.

Geologic range:-Pliocene.

\title{
Anisocorbula socialis (MARTIN, 1880)
}

P1. 5, Figs. 23a-b

1880. Corbula socialis MARTIN. Tertiarschichten auf Java, p. 92, pl. 15, figs. $10,10 \mathrm{a}-\mathrm{c}$.

1920. Corbula socialis. TeSch. Palaönt Von Timor, Lief. 8, p. 106, pl. 140 (22), figs. 284-286.

1971. Corbula (Anisocorbula) socialis. Sнuto, Mem. Fac. Sci., Kyushu Univ., Ser. $D$, Geol., vol. 21 , no. 1 , p. 67 , pl. 10 , figs. $7,8,9$.

Remarks:-Several rather well preserved specimens were examined. They are characterized by their small, rather thin, long shell with coarse concentric 
lines and distinct ridge running from beak to protruded posterior ventral corner. Dimensions (in $\mathrm{mm}$ ) :-

$\begin{array}{lccccr}\text { Valve } & \text { Right } & \text { Right } & \text { Right } & \text { Right } & \text { Left } \\ \text { Height } & 9.0 & 5.5 & 5.0 & 5.0 & 8.0 \\ \text { Length } & 15.5 & 9.0 & 8.5 & 8.0 & 13.5 \\ \text { Depth } & 3.5 & 2.8 & 2.0 & 2.2 & 3.0\end{array}$

Occurrence:-Loc. 5 (Common).

Recent distribution:-South east Asia.

Geologic range:-Pliocene to Recent.

Family Myochamidae

Genus Myadora GraY, 1840

Myadora ikebei HABE, 1950

Pl. 5, Fig. 24

1920. Myadora reeviana Sмiтн. Yoкоуама, Jour. Coll. Sci., Imp. Univ. Tokyo, vol. 39 , art. 6 , p. 143 , pl. 11 , figs. 12,13 .

1922. Myadora reeviana. YokoYama, Ibid., vol. 44, art. 1, p. 171, pl. 14, figs. 8, 11.

1950. Myadora ikebei HaBe. In KURodA, Illust. Cat Japan. Shells, no. 4, p. 320, pl. 4, fig. 17 .

1973. Myadora ikebei. Oyama, Palaeont. Soc. Japan, Spec. Paper, no. 17, p. 119, pl. 57 , figs. $2,3,7,8$.

Remarks:-One left and one right valves were examined. This is characterized by its small, long shell with distinct coarse concentric cords, nearly flat left valve, slightly inflated right valve and prominent beak.

Dimensions (in $\mathrm{mm}$ ):-Left valve, height 2.6, length 6.5 .

Occurrence:-Loc. 5 (Few).

Geologic distribution:-Koshiba, Naganuma and Yokosuka Formations, Kanagawa Prefecture.

Geologic range:-Pliocene to Pleistocene.

Family Cuspidariidae

Genus Cardiomya A. AdAMs, 1864

Cardiomya sp.

Remarks:-One imperfect specimen was examined. Shell thin, fragile and with distinct radial threads.

Occurrence:-Loc. 2 (Rare).

\section{References}

AkiYama, M. (1957): Amussiopecten iitomiensis (OTUKA) and its allies from Japan. Trans. Proc. Palaeont. Soc. Japan, N. S., (25), 31-39, pls. 6-7.

Aokr, N. (1960) : Molluscan fossils from the Nakazato Formation in Yokohama. Ibid., (39), 301-306, 1 pl. 
(1966) : Molluscan fossils from the Nobori Formation, Shikoku, Japan. Ibid., (62), 251-259, $1 \mathrm{pl}$.

ARAKI, Y. (1959): A Pleistocene marine fauna from near the cities of Tsu and Yokkaichi, Mie Prefecture, Southwest Japan. Ibid., (33), 19-22, 1 pl.

(1960): Geology, palaeontology and sedimentary structures (including Problematica) of the Tertiary formations developed in the environs of Tsu City, Mie Prefecture, Japan. Bull. Lib. Art. Dept., Mie Univ., Spec. Vol., (1), 3-118, pls. $1-11$.

DUNKer, W. (1882): Index Molluscorum maris japonici. 1-301, pls. 1-16.

Fischer, P. J. (1927): Beitrag zur Kenntnis der Pliozän fauna der Mollukken Inseln Seran und Obi. Paläont. v. Timor, Lief. 15, 1-179, Taf. 212-217.

FujIe, T. (1958) : Illustrated Cenozoic fossils, 28. Fossil molluscs from the Pleistocene Shishinai Formation. 1, Fossil Pelecypoda (in Japanese). Cen. Res., (28), 663679 , pls. $27-28$.

HABE, T. (1951-1953): Genera of Japanese shells. Pelecypoda, (1-3), Pelecypoda and Scaphopoda, (4), 1-326.

(1958a): Report on the Mollusca chiefly collected by the S. S. Soyo-Maru of the Imperial Fisheries Experimental Station on the continental shelf bordering Japan during the years 1922-1930. Part-3. Lamellibranchia (1). Publ. Seto Mar. Biol. Lab., 6, (3), 241-280, pls. 11-13.

(1958b) : Ditto. Part 4. Lamellibranchia (2). Ibid., 7, (1), 19-52, pls. 1-2.

(1960) : Fauna of shell bearing mollusks of the sea around Shirikishinai, Hokkaido. 1, Pelecypoda (in Japanese with English abstract). Publ. Shirikishinai Mar. Stat. Biol. Inst., (2), 1-10, pls. 1-5.

(1964a): Notes on the species of the genus Amusium (Mollusca). Bull. Nat. Sci. Mus., 7, (1), 1-5, pls. 1-2.

(1964b): Notes on the genus Cucullaea Lamarck (Mollusca). Ibid., 7, (3), 259-261.

(1977) : Systematics of Mollusca in Japan. Bivalvia and Scaphopoda (in Japanese). 1-372, pls. 1-72. Zukan-no-Hokuryukan, Tokyo.

Hanley, S. (1844): Description of New species of Cytherea. Proc. Zool. Soc., London, for 1844 , p. 110 .

HASE, K. (1967): Geology of the Alluvial plains of Miyagi Prefecture (in Japanese with English abstract). Contr. Inst. Geol. Palaeont., Tohoku Univ., (64), 1-45, pls. $1-3$.

HAtaI, K. (1941): Recent marine shell-bearing Mollusca of the South Sea Islands. (Part 1). Bull., Trop. Indust., Inst., Palau South Isl. Japan, (7), A, B, ii+160, pls. 1-79.

and Nisiyama, S. (1952): Check list of Japanese Tertiary marine Mollusca. Sci. Rep., Tohoku Univ., [2]. Spec. Vol., (3), 1-464.

HAYASAKA, S. (1960): Large-sized oysters from the Japanese Pleistocene and their paleoecological implication. Ibid., (4), 356-370, pls. 37-38.

(1961): The Geology and paleontology of the Atsumi Peninsula, Aichi Prefecture, Japan. Sci. Rep., Tohoku Univ., [2], 33, (1), 1-103, pls. 1-12.

(1969): Molluscan fauna of the Kukinaga Group in Tané-ga-shima, South Kyûshû, Japan. Rep. Fac. Sci., Kagoshima Univ., (2), 33-52, pls. 1-3.

(1973): Pliocene marine fauna from Tane-ga-shima, South Kyushu, Japan. Sci. Rep., Tohoku Univ., [2], Spec. Vol., (6), 97-108, pls. 6-7.

(1983): Cenozoic molluscan fossils from the south Kyushu and the Ryukyu Islands. Mem. Vol. Prof. OMORI, M., (Study of Molluscan Paleobiology), 357-362. and HASHimoto, S. (1970): Discovery of marine fossils from immediately below the Ata pyroclastic flow deposits, Onejime-cho, Osumi Peninsula, Kagoshima Prefecture. Rep. Fac. Sci., Kagoshima Univ., (3), 17-22, 1 pl.

and OKI, K. (1971): Note on the marine molluscan fauna from the Pleistocene 
Kogashira Formation in Kagoshima City, South Kyushu, Japan. Ibid., (4), 1-13, $1 \mathrm{pl}$.

Itoigawa, J., Shibata, H. and Nishimoto, H. (1974): Molluscan fossils of the Mizunami Group (in Japanese). Bull. Mizunami Foss. Mus., (1), 43-203, pls. 1-63.

,$--\longrightarrow$, and OKumura, Y. (1981): Miocene fossils of the Mizunami Group, central Japan. 2. Molluscs. Monogr. Mizunami Foss. Mus., (3-A), 1-53, pls. 1-52. (1981).

IWAI, T. (1959): The Pliocene deposits and molluscan fossils from the area southwest of Hirosaki City, Aomori Prefecture, Japan. Bull. Fac. Educ., Hirosaki Univ., (5), 39-61, pls. 1-2.

and Shiobara, T. (1969): Pleistocene Mollusca from Kamikita-gun, Aomori Prefecture, Japan. Ibid., (20), 1-7, pls. 1-3.

Iwasaki, Y. (1970): A Miocene molluscan fauna in the Philippines. Trans. Proc. Palaeont. Soc. Japan, N. S., (77), 205-228, 1 pl.

KAMADA, Y. (1952): On some species of Cyclina from Japan and Korea. Ibid., (8), $167-173,1 \mathrm{pl}$.

(1962): Tertiary marine Mollusca from the Joban coal-field, Japan. Palaeont. Soc. Japan, Spec. Paper, (8), 1-187, pls. 1-21.

Kanno, S. and Chang, L. (1973): Amussiopecten and its associate fauna from Northern Taiwan. Geol. Palaeont. Southeast Asia, 12, 189-206, pls. 30-31.

- OHARA, S. and CaAgusan, N. L. (1982): Molluscan Fauna from the Tartaro Formation (Upper Miocene) of Central Luzon Philippines (Contribution to the Geology and Paleontology of Southeast Asia CCXXII). 24, 51-128, pls. 14-19.

Kaseno, Y. and Matsuura, N. (1965): Pliocene shells from the Omma Formation around Kanazawa City, Japan. Sci. Rep., Kanazawa Univ., 10, (1), 27-62, pls. 1-20.

Kinoshita, T. and ISAHAYA, T. (1934): Catalogue of marine Mollusca from Hokkaido (in Japanese). Rep. Fish. Surv., Hokkaido Fish Exp. Sta., (33), 1-19, pls. 1-15.

Kuroda, T. (1929-1935): An illustrated catalogue of Japanese shells (in Japanese). Parts 1-16, Venus, vols. 1-5, append., 1-154.

(1931): Fossill Mollusca, in F. Honma, Shinano Chubu Chishitsushi (Geology of Central Shinano), Part 4 (in Japanese), 1-90, pls. 1-13.

(1933): Gastropoda and Lamellibranchiata (in Japanese). Iwanami-koza (Geology and Paleontology). 1-74.

(ed.), (1949-1953): Illustrated catalogue of Japanese shells. 1, (1-25).

and HABE, T. (1952): Check list and bibliography of the Recent marine Mollusca of Japan. 1-210.

- - - and OYAMA, K. (1971) : The sea shells of Sagami Bay. 1-741 (in Japanese), 1-489 (in English), pls. 1-121.

LISCHKE, C. E. (1869) : Japanishce Meeres-Conchylien. 1, 1-192, pls. 1-14.

Makiyama, J. (1926): Tertiary fossils from North Kankyo-do, Korea. Mem. Coll. Sci., Kyoto Imp. Univ., [B], 2, (3), 143-160, pls. 12-13.

(1927): Molluscan fauna of the lower part of Kakegawa Series in Province of Totomi. Ibid., 3, (1), 1-147, pls. 1-6.

(1932): Neogene Tertiary (in Japanese). Iwanami-koza (Geology and Paleontology), 1-57, $1 \mathrm{pl}$.

(1957-1960): Matajiro Yokoyama's Tertiary fossils from various localities in Japan. Parts. 1-4. Palaeont. Soc. Japan, Spec. Paper, (3-6), pls. 1-119.

MARTIN, K. (1879-80): Tertiarschichten auf Java nach den Entdeckungen von Fr. JUNGHUHN, 1-126, Tab. 1-24.

(1883-1887) : Paläontologische Ergebnisse von Tiefbohrungen auf Java nebst allegemeinen Studien über das Tertiär von Java, Timor und einigen andere Inseln. Samml. Geol. Reichs-Mus. Leiden, Ser. 1, 3, 1-381, Tab. 1-15.

(1909-1922) : Die Fossilien von Java. Ibid., N. F., 1, Abt. 2, (1-4), 333-538, Taf. $46-48$. 
Martini und Chemnitz (1838-1920): Systematische Conchylien Cabinet., 1-11.

Masuda, K. (1955): Miocene Mollusca from Noto Peninsula, Japan. Part 1 (1). Trans. Proc. Palaeont. Soc. Japan, N. S., (20), 175-183, 1 pl.

(1962) : Tertiary Pectinidae of Japan. Sci. Rep., Tohoku Univ., [2], 33 (2), 117-238, pls. 18-27.

(1973) : Molluscan biostratigraphy of the Japanese Neogene (in Japanese with English abstract). Mem. Geol. Soc. Japan., (8), 107-120, pls. 1-2.

(1978): Neogene Pectinidae of the Northern Pacific. The Veliger, 21, (2), 197-202.

(1980): Pliocene biostratigraphy in Japan based on pectinids. Saito Ho-on Kai Mus. Nat. Hist., Res. Bull., (48), 9-19, pls. 2-3.

(1983) : Origin and migration of Japanese Cenozoic Pectinidae (in Japanese). Origin and Migration of Japanese Cenozoic Mollusca, 1-12.

and NODA, H., (1976): Checklist and bibliography of the Tertiary and Quaternary Mollusca of Japan, 1950-1974. 1-494, Saito Ho-on Kai, Sendai.

and OGaSAwARA, K. (1981): On the Omma-Manganzi Fauna and Tatsunokuchi Fauna (in Japanese with English abstract). Mem. Vol. Prof. M. O MORI, 223-249, pls. 1-3.

Matsushima, Y. (1969): On the molluscan fossils from the Alluvial deposits in Yokohama City (in Japanese with English abstract). Bull. Kanagawa Pref. Mus., 1, (2), 79-96, pls. 10-13.

Matsumoto, H. (1930): On the marine fauna of three fossil zones of the Upper Miocene of Natori district, Province of Rikuzen. Sci. Rep., Tohoku Imp. Univ., [2], 13, (3), 95-109, pls. 39-40.

NodA, H. (1965): Some fossil Anadara from Southwest Japan. Trans. Proc. Palaeont. Soc. Japan, N. S., (59), 92-109, pls. 10-11.

(1966): The Cenozoic Arcidae of Japan. Sci. Rep., Tohoku Univ., [2], 38, (1), 1-161, pls. 1-14.

(1971): New anadarids associated molluscan fauna from the Haneji Formation, Okinawa-jima, Ryukyu Islands. Trans. Proc. Palaeont., Soc. Japan, N. S., (81), 27-51, pls. 6-7.

(1973): Additional notes on the Miyagian marine fauna from the Gobanshoyama Formation, Ojika Peninsula, Miyagi Prefecture, Japan. Saito Ho-on Kai Mus. Nat. Hist., Res. Bull., (42), 31-45, pls. 4-5.

(1980): Molluscan fossils from the Ryukyu Islands, Southwestern Japan. Part-1. Gastropoda and Pelecypoda from the Shinzato Formation in southeastern part of Okinawa-jima. Sci. Rep. Inst. Geosci., Univ., Tsukuba, Sec. B, 1, 1-95, pls. 1-12.

Nomura, S. (1933): Catalogue of the Tertiary and Quaternary Mollusca from the Island of Taiwan (Formosa) in the Institute of Geology and Paleontology, Tohoku Imperial University, Sendai, Japan. Part 1, Pelecypoda. Sci. Rep., Tohoku Imp. Univ., [2], 16, (1), 1-108, pls. 1-4.

(1937): The molluscan fauna from the Pliocene of Tosa. Japan. Jour. Geol. Geogr., 14, (3-4), 67-90, 1 pl.

(1938): Molluscan fossils from the Tatunokuti shell bed exposed at Goroku cliff in the western border of Sendai. Sci. Rep. Tohoku Imp. Univ., [2], 19, (2), 235275, pls. 33-36.

and HAtaI, K. (1937): A list of the Miocene Mollusca and Brachipoda collected from the region lying north of the Nanakita river in the vicinity of Sendai Rikuzen Province, Japan. Saito Ho-on Kai Mus., Res. Bull., (13), 121-145, pls. 17-21.

and Ninno, H. (1932): Fossil Mollusca from Izu and Hakone. Sci. Rep., Tohoku Imp. Univ., [2], 15, (3), 169-192, pls. 11-12.

and ONISI, H. (1940): Neogene Mollusca from the Sennan district, Miyagi Prefecture, Japan. Japan. Jour. Geol. Geogr., 17, (3-4), 181-194, pls. 17-19. 
and Zinbo, N. (1934) : Marine Mollusca from the "Ryukyu Limestone" of Kikaizima, Ryukyu Group. Sci. Rep., Tohoku Imp. Univ., [2], 16, (2), 109-164, 1 pl.

Oкамото, K. (1978) : Pleontological note of megafossils. In Honza, E. (ed), Geological investigation in the northern margin of Okinawa Trough and Western margin of Japan Sea. Geol. Surv. Japan, Cruise Rep., (10), 55-58.

and HoNZA, E. (1978): The "Pliocene" fossil molluscan assemblage including Amussiopecten collected GH-77-2 cruise in the southwestern Japan Sea. Jour. Geol. Soc. Japan, 84, (10), 625-628.

OTUKA, Y. (1935): The Oti Graben in southern Noto Peninsula, Japan. Part 3. Bull. Earthq. Res. Inst., 13, pt. 4, 846-909, pls. 53-57.

(1938) : Neogene fossils of the Ihara district, Sizuoka Prefecture, Japan. Jour. Fac. Sci., Imp. Univ. Tokyo, Sec. 2, 5, pt. 1, 1-19, pls. 1-2.

Oyama, K. (1950) : Remarks on Japanese fossil molluscan name. Part 1, Pelecypoda (in Japanese). Mineral. Geol., 3, (6), 225-228.

(1973): Revision of Matajiro Yokoyama's type Mollusca from the Tertiary and Quaternary of the Kanto area. Palaeont. Soc. Japan, Spec. Paper, (17), 1148 , pls. $1-57$.

und SAKA, K. (1944): Fossilen mollusken der Tukiyosi-Formation (1) (in Japanese with German abstract). Bull. Shigenkagaku Kenkyujo, 1, (2), 137-144, pls. 14-15.

OzAKI, H. (1958): Stratigraphical and paleontological studies on the Neogene and Pleistocene formations of the Tyosi district. Bull. Nat. Sci. Mus., 4, (1), 1-182, pls. 1-24.

, Fukuta, O. and ANdo, Y. (1954) : List of fossil molluscs from Tokumaru shell bed of the Pleistocene Tokyo Formation (in Japanese with English resume). Ibid., 3, (3), 162-175, 25-33.

Prashad, B. (1932): The Lamellibranchia of the Siboga Expedition. Systematic part, II, Pelecypoda. Uitkomsten Siboga Monogr. 53C.

ReEve, L. A. (1843-1865): Conchologia Iconica, 1-15. (1863-1864): Ibid. 14, Venus, 1-141, pls. 1-24.

RöMER, E. (1869) : Monographie der Mollusken Gattung Venus LinNe. Bd. 1, Cytherea LAMARCK, 1-217, pls. 1-59.

Schenck, H. G. (1936): Nuculid bivalves of the Genus Acila. Geol. Soc. Amer., Spec. Paper, (4), 1-149, pl. 1-18.

Sнuтo, T. (1955): Amussiopecten from Miyazaki Group, Miyazaki Prefecture, Japan, (Palaeontological study of the Miyazaki Group-2). Trans. Proc. Palaeont. Soc. Japan, N. S., (20), 101-110, pls. 16-17.

(1957a): Fossil Paphia from Miyazaki Group. (Palaeontological study of the Miyazaki Group-III). Japan. Jour. Geol. Geogr., 28, (1-3), 139-160, pl. 1. (1957b) : Crassatellites and Venericardia from the Miyazaki Group. (Palaeotological study of the Miyazaki Group-VII). Mem. Fac. Sci., Kyushu Univ., [D], 6, (2), 69-80, $1 \mathrm{pl}$.

(1957c): Polymorphism in mollusks and facies differentiation. (Palaeontological basis for the consideration of speciation-1) (in Japanese with English abstract). Jour. Geol. Soc. Japan, 63, (745), 565-585.

(1960) : On some pectinids and venerids from the Miyazaki Group. (Palaeontological study of the Miyazaki Group-VII). Mem. Fac. Sci., Kyushu Univ., [D], 9, (3), 119-149, pls. 12-14.

(1961): Palaeontological study of the Miyazaki Group. A General account of the faunas. Ibid. 10, (2), 73-206, pls. 11-13.

(1971): Neogene Bivalves from Panay Island, the Philippines (Contributions to the Geology and Palaeontology of South east Asia, CVII). Mem. Fac. Sci., Kyushu Univ., [D], 21, (1), 1-73, pls. 1-10.

Sмiтh, W. D. (1913): Contributions to stratigraphy and fossil invertebrate fauna of the Philippine Islands. Philip. Jour. Sci., 8, (4), Sec. A, 235-300, pls. 1-20. 
Sowerby, G. B. (1842-1887) : Thesaurus Conchyliorum, vols. 1-5.

TAKI, I. and OYama, K. (1954): Matajiro Yokoyama's the Pliocene and later faunas from the Kwanto region in Japan. Palaeont. Soc. Japan, Spec. Papers. (2), $1-68$, pls. $1-49$.

Tesch, P. (1920): Jungtertiäre und Quartäre Mollusken von Timor. Paläeont $v$. Timor, Zweit. Teil. Life. 8, 41-121, Taf. 129-140.

TokUnaga, S. (1906) : Fossils from the environs of Tokyo. Jour. Coll. Sci., Imp. Univ. Tokyo, 21, art. 2, 1-96, pls. 1-6.

WAKIYAMA, Y. (1929): Japanese food oysters. Japan. Jour. Zool., 22, (3), 359-367, pls. 8-10.

YaBE, H. and HataI, K. (1942) : Additional fossils from the Shimaziri beds of Okinawazima, Ryukyu Islands, Japan. Japan. Jour. Geol. Geogr., 18, (1-2), 71-78, 1 pl.

YAMADA, J. (1963): Remarks on the significance of the Pleistocene Mollusca from the Shima Peninsula, Mie Prefecture, Japan. Bull. Fac. Art. Sci., Mie Univ., (27), 96-103, 1 pl.

Yamamoto, G. and HaBe, T. (1958): Fauna of shell-bearing molluses in the Mutsu Bay. Lamellibranchia (1). Bull. Mar. Biol. Stat. Asamushi, Tohoku Univ., 9, (1), 1-20, pls. 1-5.

Yokoyama, M. (1920): Fossils from the Miura Peninsula and its immediate north. Jour. Coll. Sci., Imp. Univ. Tokyo, 39, art. 6, 1-193, pls. 1-20.

(1922): On a new species of Pecten from the Neogene of Japan. Jour. Geol. Soc. Tokyo 29, (350), 1-2, 1 pl.

(1923): Tertiary Mollusca from Dainichi in Totomi. Jour. Coll. Sci., Imp. Univ. Tokyo, 45, art. 2, 1-18, pls. 1-2.

(1926): Tertiary Mollusca from Southern Totomi. Jour. Fac. Sci. Imp. Univ. Tokyo, Sec. 2, 1, pt. 9, 313-364.

(1928a): Shells from Hyuga. Ibid., 2, pt. 7, 331-350, pls. 66-67.

- (1928b) : Mollusca from oil-field of the Islands of Taiwan. Imp. Geol. Surv. Japan, Rep., (101), 1-112, pls. 1-18.

(1929): Pliocene shells from Tonohama, Tosa. Ibid., (104), 9-17, pls. 7-8. 